
Not for reproduction, distribution or commercial use.

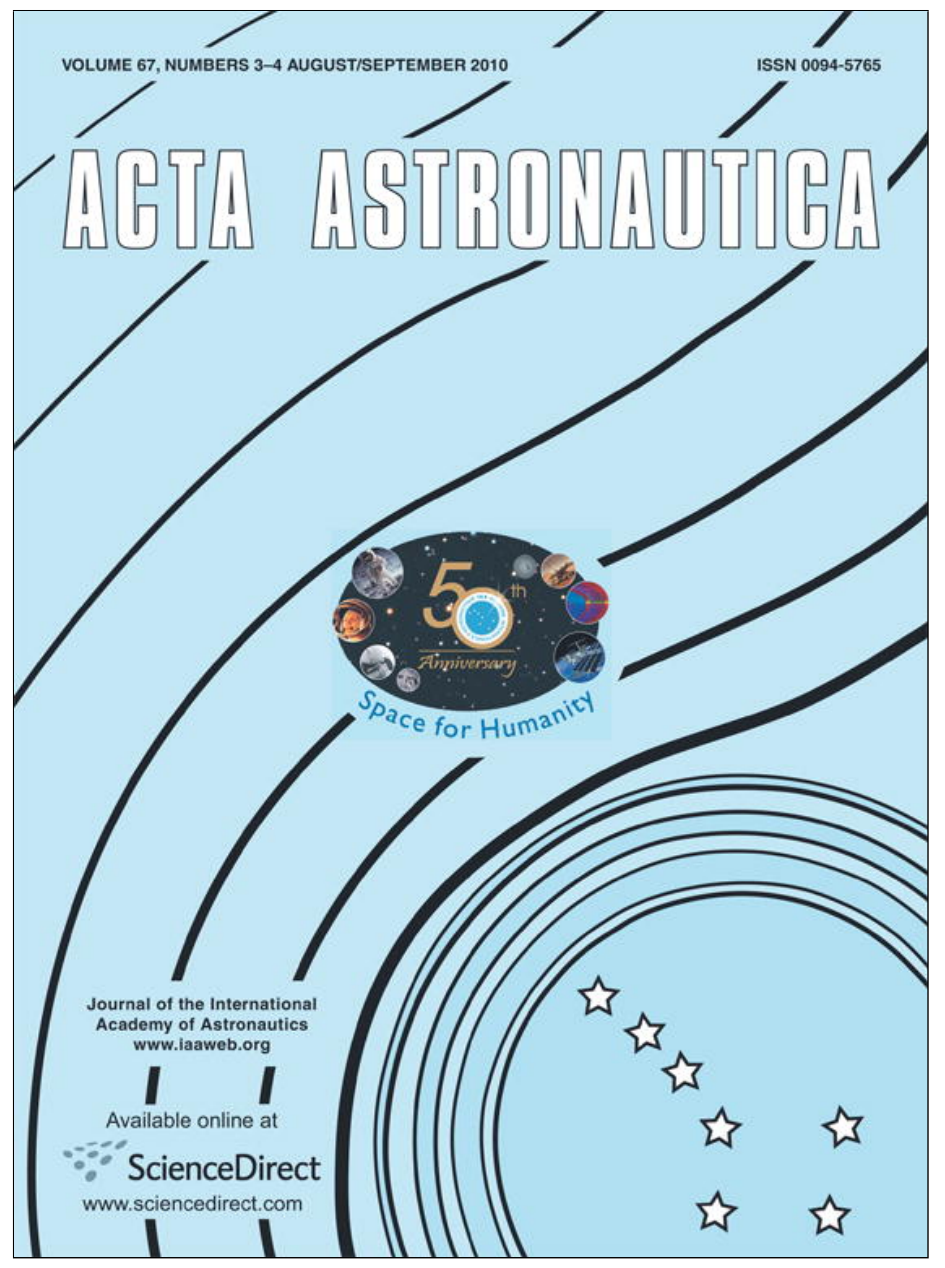

This article appeared in a journal published by Elsevier. The attached copy is furnished to the author for internal non-commercial research and education use, including for instruction at the authors institution and sharing with colleagues.

Other uses, including reproduction and distribution, or selling or licensing copies, or posting to personal, institutional or third party websites are prohibited.

In most cases authors are permitted to post their version of the article (e.g. in Word or Tex form) to their personal website or institutional repository. Authors requiring further information regarding Elsevier's archiving and manuscript policies are encouraged to visit:

http://www.elsevier.com/copyright 


\title{
Explicit solution to the full nonlinear problem for satellite formation-keeping
}

\author{
Hancheol Cho ${ }^{\mathrm{a}, *}$, Firdaus E. Udwadia ${ }^{\mathrm{a}, \mathrm{b}, \mathrm{c}, \mathrm{d}, \mathrm{e}}$ \\ a Department of Aerospace and Mechanical Engineering, University of Southern California, 430K Olin Hall, Los Angeles, CA 90089, USA \\ ${ }^{\mathrm{b}}$ Department of Civil Engineering, University of Southern California, 430K Olin Hall, Los Angeles, CA 90089, USA \\ ${ }^{\mathrm{c}}$ Department of Mathematics, University of Southern California, 430K Olin Hall, Los Angeles, CA 90089, USA \\ d Department of Systems Architecture Engineering, University of Southern California, 430K Olin Hall, Los Angeles, CA 90089, USA \\ e Department of Information and Operations Management, University of Southern California, 430K Olin Hall, Los Angeles, CA 90089, USA
}

\section{A R T I C L E I N F O}

\section{Article history:}

Received 26 August 2009

Received in revised form

1 December 2009

Accepted 11 February 2010

Available online 5 March 2010

Keywords:

Satellite formation-keeping

Udwadia-Kalaba equation

Hill frame

Nonlinear control

\begin{abstract}
A B S T R A C T
This paper presents simple and exact formation-keeping guidance schemes that use a new method that is rooted in some recent advances in analytical dynamics. As a result of this new approach, explicit control inputs to exactly maintain a given formation configuration are easily determined using continuous thrust propulsion systems. The complete nonlinear problem is addressed, and no linearizations and/or approximations are made. The approach provides a marked improvement over existing results in that the control forces, which cause geometric formation-keeping constraints to be exactly satisfied for arbitrary reference orbits, are found in closed form. For Keplerian reference orbits, a much simpler and explicit expression for the control needed to exactly satisfy formation-keeping constraints than hereto available is obtained. The paper also includes explicit control results when the follower is inserted into orbit with incorrect initial conditions, as usually happens in practice. The Hill reference frame, which is often more intuitive for formation-keeping, is used in the analysis. While this paper takes an example of a projected circular formation, the methodology that is developed can be applied to any desired configuration or orbital requirements. Extensive computational simulations are performed to demonstrate the ease of implementation, and the numerical accuracy provided by the approach developed herein.
\end{abstract}

(c) 2010 Elsevier Ltd. All rights reserved.

\section{Introduction}

Over the past few years, formation flying has received much attention from the aeronautical and astronautical community as a new concept to overcome the demerits of conventional space technologies. By using small, multiple satellites, total mission costs can be substantially reduced and missions made more efficient and flexible. Also, the use of fleets of small satellites flying in formations, instead of a single monolithic satellite, enables many applications

\footnotetext{
* Corresponding author. Tel.: +1213 703 1613; fax: +12137408071.

E-mail addresses: hancheoc@usc.edu, hancheol.cho@gmail.com (H. Cho).
}

such as optical interferometry, distributed sensing, gravitational field measurements, ionospheric observation, Earth observation, and 3-D mapping for planetary explorers. In this paper, a new formation-keeping technique is proposed for formation flying. This control problem typically occurs when distributed arrays of multiple satellites are used to form virtual apertures. To successfully achieve mission goals related to interferometric measurements, for example, it is very important to maintain the desired aperture's size at all times. In the formation-keeping the relative motion between the satellites is referenced with respect to one satellite, called the leader. The other satellites whose relative motion are of interest, are designated as the followers. In this paper, it is assumed that the leader, whose orbit is referred to as 
the reference orbit, is passive, i.e., non-maneuvering, and only the follower satellites are controlled in order to meet the desired formation-keeping goals.

We next provide a brief overview of the state-of-theart in formation-keeping so as to point out the salient differences between the approach presented herein and the approaches that have so far been investigated. Scharf et al. $[1,2]$ provide a rigorous survey of satellite formationflying guidance and control, and a brief history of several important techniques in formation flying, for example, path planning, formation-keeping, optimal controller design, and stability analysis, are introduced. While several control strategies for formation-keeping have been developed by various researchers to date, much of the research focuses on the design of passive apertures, which are periodic configurations of satellite formations with proper initial conditions, while reducing the tendency of the satellites to drift apart due to various perturbations [1-3]. Designing these passive apertures requires an analysis of the relative motion of satellites in two close, neighboring orbits, and linearized equations such as the Hill-Clohessy-Wiltshire (HCW) equations for circular reference orbits [4,5] and the Tschauner-Hempel (TH) equations for elliptical reference orbits [6] have been widely used due to their simplicity. Yan et al. [7] design a pulse-based controller for the satellites' periodic motion using the linear quadratic regulator (LQR) technique. They consider circular reference orbits, and the HCW equations are employed. Sparks [8] discretizes the HCW equations and uses discrete-time LQR theory to maintain a projected circular formation, which is also chosen as the desired formation geometry in this paper, in the presence of the Earth's nonspherical gravity perturbations. As in [7], a circular reference orbit is considered. Based on the $\mathrm{TH}$ equations, Inalhan et al. [9] present the initial conditions with which an array of satellites makes closed orbits around the leader as seen in the Hill frame. Their results extend the previous results to unperturbed, elliptical reference orbits. Tillerson and How [10] propose guidance algorithms for formation-keeping using linear programming (LP). Their LP approach is based on three linearized equations of relative motion, including the HCW equations for unperturbed, circular reference orbits, modified HCW equations for $J_{2}$-perturbed circular reference orbits, and the TH equations for unperturbed, elliptical reference orbits. Qingsong et al. [11] design a low-thrust fuzzy controller to solve the formation-keeping problem for a circular reference orbit. Since in [7-11], the linearized equations about Keplerian reference orbits are employed, the solutions derived are suitable only for very smallsized formations wherein the distances between the leader and the followers is less than a few kilometers; the accuracy of the results are limited by the linearizations used. Using Lyapunov technique, Hadaegh et al. [12] design an adaptive controller for formation-keeping in the presence of constant, but unknown disturbances without linearizing the relative dynamics. Milam et al. [13] find an optimal control method to maintain a relative geometry. Their procedure is essentially numerical and they use their own numerical solver, called the nonlinear trajectory generation (NTG) software package. No et al. [14] use power series and trigonometric functions to get closedform relative equations of motion (between the satellites) from general nonlinear dynamics. A series of impulsive maneuvers is numerically obtained to maintain a formation configuration that lies within a certain prescribed spatial region.

Thus we see that previous work mainly focuses on linearized approaches to the formation-keeping problem in which the trajectory of the leader is taken to be a simple circular or elliptical Keplerian orbit, because it is then easy to design controllers based on traditional linear control techniques. For arbitrary reference orbits, the controllers for formation-keeping to date have been obtained in complicated ways that only lend themselves to numerical approaches. With the increasing complexity of future space missions and the need for much higher accuracies in formation-keeping, however, leader satellites will be required to fly along arbitrarily prescribed trajectories and the complete nonlinear equations will need to be included to maintain the desired accuracies.

This paper does away with linearizations and approximations and obtains exact, analytical solutions to the general formation-keeping problem in which the reference orbit of the leader can be any (suitably smooth) arbitrarily prescribed function of time. The closed-form solutions for the control forces to be applied to the follower(s) developed herein do not necessitate complex, time-consuming numerical procedures. They are extremely useful since they provide deeper analytical insights into the system's dynamics and control, enabling assessments such as detailed sensitivity analyses. They have an even more important practical utility since they substantially reduce computational loads thereby making possible on-orbit computations in real-time.

Our methodology is based on a recent, new approach to explicitly formulate the equations of motion for constrained systems proposed by Udwadia and Kalaba [15-21]. Based on Gauss's principle [22], they presented an analytical method to solve the problem of highly constrained motion even when many non-integrable constraints are included. Also, this approach does not distinguish between holonomic or nonholonomic constraints unlike the traditional methods such as when using Lagrange multipliers. Due to simplicity and generality, many researchers have used this method to solve problems in this field of study. Schutte and Dooley [23] use this method to study the periodicity of tethered satellite motion and Lam [24] determines the control force needed to cause a satellite to move at a fixed inclination in a circular orbit even when the Earth oblateness gravity perturbation is considered. Lam et al. [25] obtain the deterministic control force to maintain the formation configurations when the formation is around a non-spherical spinning Mars. The initial conditions used are the ones that exactly satisfy the given configuration constraints and the problem is solved in the inertial coordinate frame of reference. Cho and Yu [26] obtain a solution to the formation-keeping problem of multiple satellites circling a spherical Earth. They constrain the relative distances of the formation in the Hill frame, and also use the Udwadia-Kalaba approach. They solve the 
problem in the inertial frame first, and then transform to the Hill frame for better visualization. Though they do not use any linearization, their reference orbit is limited to an unperturbed, circular orbit, and they use initial conditions that exactly fulfill their formation-keeping requirements.

The purpose of this paper is to present explicit and exact formation-keeping guidance schemes that determine control inputs and state trajectories directly in the Hill frame. For the follower satellites we choose the projected circular orbit (PCO) in which the relative orbit is a circle when projected onto the local horizontal plane (see Fig. 1). This geometry has significant practical utility. Besides being rigorously investigated in many papers $[8,10,27]$, it was planned [14] to be used for the TechSat-21 mission which was, unfortunately, cancelled later on. We assume unbounded continuous burn throughout the maneuvers and place no restrictions on the leader's reference orbit. That is, the reference orbit can be an arbitrary function of time to include the effects of various disturbances and/or thrust. We extend the practical utility of the exact, explicit closed-form solutions obtained herein, to include a method to correct initial-condition misalignment problems, since in practice there is no guarantee that the follower satellites are initially inserted exactly into the desired orbits. Once the reference orbit is provided, one can immediately generate the necessary control forces and state trajectories to satisfy the given constraints on the relative (Hill frame) geometry. Since the problem is solved in the Hill frame, it lends itself to a better intuitive understanding. Extensive numerical simulations are performed to show the simplicity of the approach developed herein, the effectiveness of the control strategy generated, the ease of its computational implementation, and the accuracy of the numerical results. We also show that the general formulation that deals with an arbitrarily prescribed reference orbit when particularized to a Keplerian elliptical orbit yields the expected results.

The paper is organized in the following manner. In Section 2, we briefly discuss the fundamental equation of

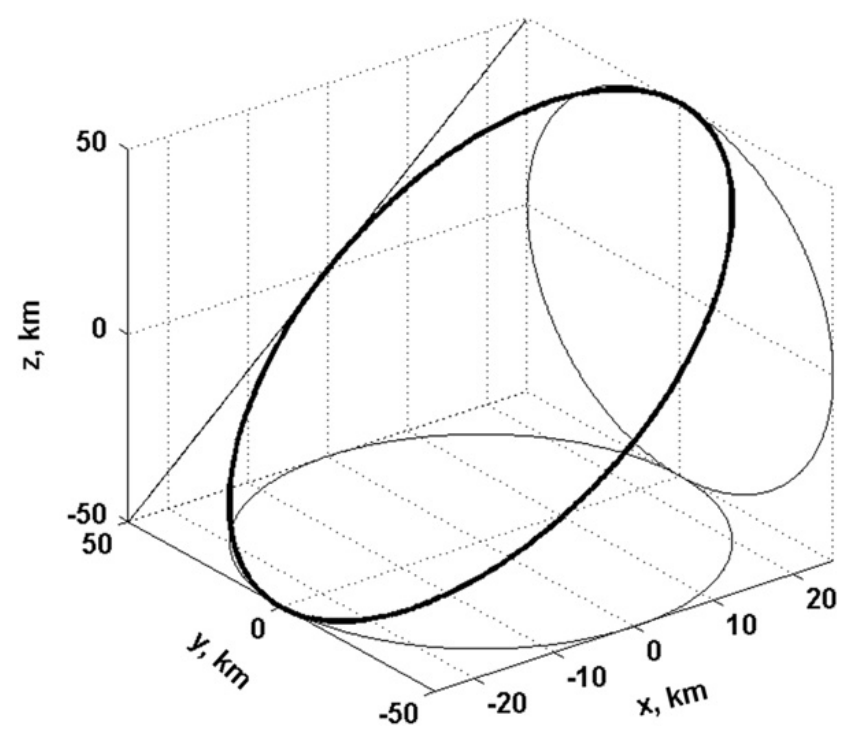

Fig. 1. Projected circular orbit (PCO) in the Hill frame. motion for constrained systems, the Udwadia-Kalaba equation. Explicit, closed-form expressions for the control forces are presented. Section 3 deals with the so-called unconstrained motion that is derived from Newton's law of gravitation expressed in the Hill frame. In Section 4, the two constraints for PCO are introduced, and constrained motion in the Hill frame is handled in two ways depending upon the reference orbit. General reference orbits and their particularization to Keplerian orbits are considered. In Section 5, if the initial conditions are not correct for the followers, the new constraint equations are derived. Finally, in Section 6 numerical simulations are presented to demonstrate the efficacy and the accuracy of the closed-form method.

\section{Udwadia-Kalaba equation}

This section deals with the fundamental equation, called the Udwadia-Kalaba equation, for constrained systems. We consider $N$ point-mass particles (or satellites in this paper) in generalized coordinates. It is assumed that the initial position and velocity of the particles are known and that the generalized displacement vector and generalized velocity vector are denoted by $\mathbf{x}(t)$ and $\dot{\mathbf{x}}(t)$ respectively, where

$\mathbf{x}(t)=\left[\begin{array}{llll}x_{1} & x_{2} & \ldots & x_{n}\end{array}\right]^{\mathbf{T}}$,

and $t$ represents time, the superscript "T" denotes the transpose of a vector or a matrix, and $n$ is the number of generalized coordinates. If the forces impressed on the particles are denoted by

$\mathbf{F}(t)=\left[\begin{array}{llll}F_{1}(t) & F_{2}(t) & \ldots & F_{n}(t)\end{array}\right]^{\mathbf{T}}$,

the Lagrange equation for the unconstrained motion of the system can now be expressed as an $n$ by 1 matrix equation

$\mathbf{M} \ddot{\mathbf{x}}(t)=\mathbf{F}[\mathbf{x}(t), \dot{\mathbf{x}}(t), t]$,

or

$\ddot{\mathbf{x}}(t)=\mathbf{M}^{-1} \mathbf{F}[\mathbf{x}(t), \dot{\mathbf{x}}(t), t] \triangleq \mathbf{a}(t)$,

where $M>0$ is the $n$ by $n$ mass matrix. We denote by $\mathbf{a}(t)$ the unconstrained acceleration of the system at the time $t$.

Now $p$ constraints are imposed on this system that is described by Eqs. (3) and (4). They are of the form

$\varphi_{j}(\mathbf{x}, \dot{\mathbf{x}}, t)=0, j=1,2, \ldots, p$.

It should be noted that the constraint of Eq. (5) can either be holonomic or nonholonomic, and time dependent. Unlike previous research, the fundamental equation that we will discuss hereafter handles both constraints with equal ease. By differentiating Eq. (5) with respect to time once (for nonholonomic constraints) or twice (for holonomic constraints), we get the following constraint equation of the form

$\mathbf{A}(\mathbf{x}(t), \dot{\mathbf{x}}(t), t) \ddot{\mathbf{x}}=\mathbf{b}(\mathbf{x}(t), \dot{\mathbf{x}}(t), t)$,

where the matrix $\mathbf{A}$ is $p$ by $n$ and $\mathbf{b}$ is an $p$ by 1 vector. The presence of the constraint Eq. (6) causes additional forces 
of constraint $\mathbf{F}^{\mathbf{C}}(t)$ to be applied to the particles and the resulting equation of motion becomes

$\mathbf{M} \ddot{\mathbf{x}}(t)=\mathbf{F}[\mathbf{x}(t), \dot{\mathbf{x}}(t), t]+\mathbf{F}^{\mathbf{C}}(t)$.

Our task then is the determination of the constraint force $\mathbf{F}^{\mathbf{C}}(t)$. Based on the Gauss's principle of least constraint [22], Udwadia and Kalaba [28] proposed that the relation for the constraint force vector is explicitly given by

$\mathbf{F}^{\mathbf{C}}(t)=\mathbf{M}^{1 / 2}\left(\mathbf{A M}^{-1 / 2}\right)^{+}(\mathbf{b}-\mathbf{A a})$,

where the superscript "+" represents the Moore-Penrose generalized inverse.

Now from Eqs. (7) and (8), we have the following equation of motion of the constrained system

$\mathbf{M} \ddot{\mathbf{x}}=\mathbf{M a}+\mathbf{M}^{1 / 2}\left(\mathbf{A} \mathbf{M}^{-1 / 2}\right)^{+}(\mathbf{b}-\mathbf{A a})$,

or

$\ddot{\mathbf{x}}=\mathbf{a}+\mathbf{M}^{-1 / 2}\left(\mathbf{A} \mathbf{M}^{-1 / 2}\right)^{+}(\mathbf{b}-\mathbf{A a})$.

In this paper, Eq. (10) is referred to as the UdwadiaKalaba equation. The constraint force vector $\mathbf{F}^{\mathbf{C}}(t)$ in Eq. (8), enables all the constraints to be exactly satisfied at every instant of time. In what follows, this constraint force will be the control force that we shall use to ensure that the followers exactly satisfy their formation-keeping requirements.

If we are concerned with only one particle in a Cartesian inertial frame of reference, things can be much simpler. Using the fact that the mass matrix $\mathbf{M}$ is $\mathbf{M}=m \mathbf{I}_{3 \times 3}$ where $\mathbf{I}_{3 \times 3}$ is the 3 by 3 identity matrix and $m$ is the mass of the particle, it can be shown that the Udwadia-Kalaba equation becomes [28]

$\ddot{\mathbf{x}}=\mathbf{a}+\mathbf{A}^{+}(\mathbf{b}-\mathbf{A a})$,

so that

$m \ddot{\mathbf{x}}=m \mathbf{a}+m \mathbf{A}^{+}(\mathbf{b}-\mathbf{A a})$,

and the control (constraint) force $\mathbf{F}^{\mathbf{C}}$ is explicitly found as $\mathbf{F}^{\mathbf{C}}=m \mathbf{A}^{+}(\mathbf{b}-\mathbf{A a})$.

In what follows we shall use the terms "unconstrained motion" to mean "uncontrolled motion"; the terms "constrained motion" to mean "controlled motion"; and the terms "constraint force" to mean "control force."

It should be noted that the force $\mathbf{F}^{\mathbf{C}}(t)$ in Eq. (8) or (13) minimizes the cost $J(t)=\left(\mathbf{F}^{\mathbf{C}}\right)^{\mathbf{T}} \mathbf{M}^{-1} \mathbf{F}^{\mathbf{C}}$ at each instant of time among all the control forces that are compatible with the given constraints. While for simplicity, in this paper, we have used the weighting matrix to be the matrix $\mathbf{M}^{-1}$ in the cost function $J(t)$, any other positive definite weighting matrix $\mathbf{W}$ could have been chosen (see [21] for details).

\section{Uncontrolled motion}

Before adding a control (constraint) force to the system, we must specify the uncontrolled (unconstrained) motion that describes the natural behavior of a particle or a satellite. The leader and the follower satellites move only under the influence of gravity, and hence, as stated before, we consider "unconstrained" motion and "uncon- trolled" motion to have the same meaning. In the case of constrained motion, proper control forces are needed to be applied on the unconstrained system to meet the constraint requirements. Hence, we consider "constrained" motion and "controlled" motion to also have the same meaning.

The motion of a satellite orbiting around the Earth is governed by Newton's law of gravitation [29] so that

$\mathbf{a}=\left[\begin{array}{c}\ddot{X} \\ \ddot{Y} \\ \ddot{Z}\end{array}\right]=-\frac{G M_{\oplus}}{\left(X^{2}+Y^{2}+Z^{2}\right)^{3 / 2}}\left[\begin{array}{c}X \\ Y \\ Z\end{array}\right]$,

where $G$ is the universal gravitational constant and $M_{\oplus}$ is the mass of the Earth. Eq. (14) represents the unconstrained acceleration vector like Eq. (4). It must be noted that Eq. (14) is described in the inertial frame, more precisely the ECI (Earth-Centered Inertial) frame [30]. This coordinate frame originates at the center of the Earth, the $X$ axis points towards the vernal equinox, the $Y$ axis is $90^{\circ}$ to the east in the equatorial plane, and the $Z$ axis extends through the North Pole (see Fig. 2).

As seen later, it is convenient to define a new coordinate frame, called the Hill frame. The origin of this frame is located at the leader satellite that moves in the reference orbit, the $x$-axis is directed radially outward along the local vertical, the $z$-axis is along the angular momentum vector, and the $y$-axis is taken perpendicular to the $x z$-plane so as to form a right-handed triad (see Fig. 2). Since our aim is to handle the problem in the Hill frame, it is necessary to describe Eq. (14) in this frame by using the transformation between the ECI frame and the Hill frame. In general, these two coordinate frames have the following relationship

$\left[\begin{array}{c}x \\ y \\ z\end{array}\right]=\mathbf{R}\left[\begin{array}{c}X \\ Y \\ Z\end{array}\right]-\left[\begin{array}{c}r_{L} \\ 0 \\ 0\end{array}\right]$,

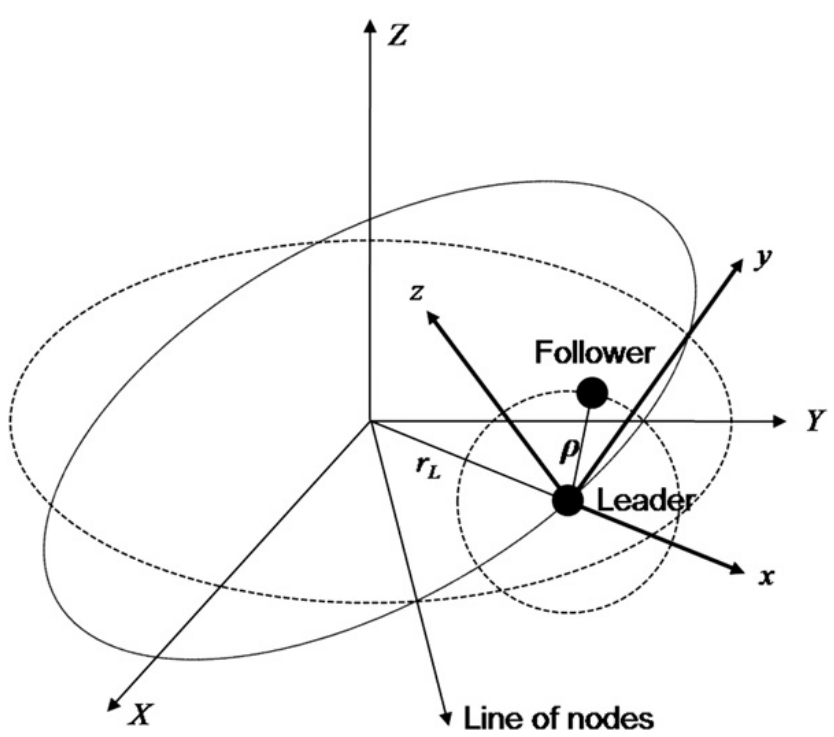

Fig. 2. The ECI frame $(X-Y-Z)$ and the Hill frame $(x-y-z)$. For simplicity, the leader is shown to follow an elliptical orbit. The general methodology presented in the paper, however, is valid for arbitrarily prescribed trajectories of the leader. 
or

$\left[\begin{array}{c}X \\ Y \\ Z\end{array}\right]=\mathbf{R}^{-1}\left[\begin{array}{c}x+r_{L} \\ y \\ z\end{array}\right]=\mathbf{R}^{\mathbf{T}}\left[\begin{array}{c}x+r_{L} \\ y \\ z\end{array}\right]=\mathbf{S}\left[\begin{array}{c}x+r_{L} \\ y \\ z\end{array}\right]$

where $\left[\begin{array}{lll}x & y & z\end{array}\right]^{\mathbf{T}}$ denotes the coordinates of an arbitrary point in the Hill coordinate frame, $\left[\begin{array}{lll}X & Y & Z\end{array}\right]^{\mathbf{T}}$ denotes its coordinates in the ECI coordinate frame, $\mathbf{R}$ is an orthogonal rotation matrix, $\mathbf{S}$ is the transpose of $\mathbf{R}$, and $r_{L}$ (the subscript " $L$ " denotes quantities pertinent to the leader) is the distance between the origin of the ECI frame and the origin of the Hill frame (leader). In Eq. (15), we translate the rotated ECI system by $-r_{L}$ along the $x$-axis to have its origin coincide with the leader's position, namely with the origin of the Hill frame. Obtaining the proper rotation matrix $\mathbf{R}$ will be the main task in the next section. Eqs. (15) and (16) can be written more succinctly as follows

$\mathbf{X}=\mathbf{R} \mathbf{X}-\boldsymbol{\gamma}$,

and

$\mathbf{X}=\mathbf{S} \mathbf{x}+\mathbf{S} \boldsymbol{\gamma}=\mathbf{S} \mathbf{x}+\mathbf{w}$

where $\mathbf{X} \triangleq\left[\begin{array}{lll}x & y & z\end{array}\right]^{\mathbf{T}}, \mathbf{X} \triangleq\left[\begin{array}{lll}X & Y & Z\end{array}\right]^{\mathbf{T}}, \boldsymbol{\gamma} \triangleq\left[\begin{array}{lll}\mathrm{r}_{\mathrm{L}} & 0 & 0\end{array}\right]^{\mathbf{T}}$, and $\mathbf{w} \triangleq \mathbf{S} \boldsymbol{\gamma}$.

To get the uncontrolled (unconstrained) acceleration $\mathbf{a}$ in the Hill frame, we start from the Lagrange equation

$\frac{d}{d t}\left(\frac{\partial T}{\partial \dot{\mathbf{x}}}\right)-\frac{\partial T}{\partial \mathbf{x}}=\mathbf{Q}(\mathbf{x}, \dot{\mathbf{x}}, t)$

where $T$ is the kinetic energy of the system and $\mathbf{Q}(\mathbf{x}, \dot{\mathbf{x}}, t)$ denotes the generalized force of the system which includes the potential energy. Since the kinetic energy $T$ must be represented in the inertial frame, it follows that

$$
\begin{aligned}
T= & \frac{1}{2} \dot{\mathbf{X}}^{\mathbf{T}} \mathbf{M} \dot{\mathbf{X}}=\frac{1}{2}\left(\mathbf{x}^{\mathbf{T}} \dot{\mathbf{S}}^{\mathbf{T}} \mathbf{M} \dot{\mathbf{S}} \mathbf{x}+\dot{\mathbf{X}}^{\mathbf{T}} \mathbf{S}^{\mathbf{T}} \mathbf{M S} \dot{\mathbf{X}}+\dot{\mathbf{w}}^{\mathbf{T}} \mathbf{M} \dot{\mathbf{w}}\right)+\left(\mathbf{x}^{\mathbf{T}} \dot{\mathbf{S}}^{\mathbf{T}} \mathbf{M S} \dot{\mathbf{X}}\right. \\
& \left.+\dot{\mathbf{w}}^{\mathbf{T}} \mathbf{M} \dot{\mathbf{S}} \mathbf{x}+\dot{\mathbf{w}}^{\mathbf{T}} \mathbf{M S} \dot{\mathbf{X}}\right),
\end{aligned}
$$

where Eq. (18) has been used. Inserting Eq. (20) into (19), we get the following equation of motion in the Hill frame

$\mathbf{S}^{\mathbf{T}} \mathbf{M S} \ddot{\mathbf{X}}=-2 \mathbf{S}^{\mathbf{T}} \mathbf{M} \dot{\mathbf{S}} \dot{\mathbf{x}}-\mathbf{S}^{\mathbf{T}} \mathbf{M} \ddot{\mathbf{S}} \mathbf{X}-\mathbf{S}^{\mathbf{T}} \mathbf{M} \ddot{\mathbf{w}}+\mathbf{S}^{\mathbf{T}} \mathbf{F}(\mathbf{X}, \dot{\mathbf{X}}, t)$,

where $\mathbf{F}(\mathbf{X}, \dot{\mathbf{X}}, t)$ is given by

$\mathbf{F}(\mathbf{X}, \dot{\mathbf{X}}, t)=\mathbf{F}(\mathbf{X})=-\frac{G M_{\oplus}}{\left(\mathbf{X}^{\mathbf{T}} \mathbf{X}\right)^{3 / 2}} \mathbf{M X}$

From Eq. (21), the matrix $\mathbf{S}^{\mathbf{T}} \mathbf{M S}$ serves as the mass matrix in the Hill frame. However, the matrix $\mathbf{M}$ is $\mathbf{M}=m \mathbf{I}_{3 \times 3}$ for each particle (or satellite) where $m$ is the mass of the particle (or satellite), so $\mathbf{S}^{\mathbf{T}} \mathbf{M S}$ is just equal to $\mathbf{M}$ owing to the orthogonality of the matrix $\mathbf{S}$. Finally, the uncontrolled acceleration vector $\mathbf{a}$ in the Hill frame can be described as

$\mathbf{a}=-2 \mathbf{S}^{\mathbf{T}} \dot{\mathbf{S}} \dot{\mathbf{x}}-\mathbf{S}^{\mathbf{T}} \ddot{\mathbf{S}} \mathbf{X}-\mathbf{S}^{\mathbf{T}} \ddot{\mathbf{w}}-\frac{G M_{\oplus}}{\left[(\mathbf{S} \mathbf{x}+\mathbf{w})^{\mathbf{T}}(\mathbf{S} \mathbf{x}+\mathbf{w})\right]^{3 / 2}}\left(\mathbf{X}+\mathbf{S}^{\mathbf{T}} \mathbf{w}\right)$ or more explicitly as

$$
\begin{aligned}
\mathbf{a}= & {\left[\begin{array}{c}
\ddot{x} \\
\ddot{y} \\
\ddot{z}
\end{array}\right]=-\left[\begin{array}{c}
\ddot{r}_{L} \\
0 \\
0
\end{array}\right]-2 \mathbf{R \dot { S }}\left[\begin{array}{c}
\dot{x}+\dot{r}_{L} \\
\dot{y} \\
\dot{z}
\end{array}\right]-\mathbf{R \ddot { S }}\left[\begin{array}{c}
x+r_{L} \\
y \\
z
\end{array}\right] } \\
& -\frac{G M_{\oplus}}{\left[\left(x+r_{L}\right)^{2}+y^{2}+z^{2}\right]^{3 / 2}}\left[\begin{array}{c}
x+r_{L} \\
y \\
z
\end{array}\right] .
\end{aligned}
$$

\section{Controlled motion}

Now we control (constrain) the motion of the follower satellites and introduce two constraints on the system. For our examples we maintain the distance between the leader and follower(s) to be a constant, say $\rho$, when projected on the $y z$-plane in the Hill frame. Also, to exclude the possibility that the motion along the $x$ coordinate diverges, another linear constraint between the $x$ and $z$ coordinates is applied. We shall assume that all the follower satellites in the formation can be continuously controlled. The strength of this paper lies in the generality and simplicity in adapting the method to other follower configurations.

In the Hill frame, the constraint equations can be written as

$2 x=z$

$y^{2}+z^{2}=\rho^{2}$,

where $\rho$ is the constant distance between the leader and the follower(s) when projected on the local horizontal $(y-z)$ plane. Eq. (25) makes the relative motion bounded for every axis, and it also matches the solutions of the HCW equations satisfying the constraint Eq. (26) [27]. Since Eqs. (25) and (26) are holonomic, the motion of the follower(s) must also satisfy the differentiated equations

$2 \dot{x}=\dot{z}$,

and

$y \dot{y}+z \dot{z}=0$.

A further differentiation with respect to time yields the following constraint equation

$\left[\begin{array}{ccc}2 & 0 & -1 \\ 0 & y & z\end{array}\right]\left[\begin{array}{c}\ddot{x} \\ \ddot{y} \\ \ddot{z}\end{array}\right]=\left[\begin{array}{c}0 \\ -\dot{y}^{2}-\dot{z}^{2}\end{array}\right]$,

which is of the form $\mathbf{A} \ddot{\mathbf{x}}=\mathbf{b}$, where

$\mathbf{A}=\left[\begin{array}{ccc}2 & 0 & -1 \\ 0 & y & z\end{array}\right], \ddot{\mathbf{x}}=\left[\begin{array}{c}\ddot{x} \\ \ddot{y} \\ \ddot{z}\end{array}\right], \mathbf{b}=\left[\begin{array}{c}0 \\ -\dot{y}^{2}-\dot{z}^{2}\end{array}\right]$.

If we consider the case wherein we have a single follower, we can use Eq. (11) directly. Since the matrix $\mathbf{A}$ is simple, we can easily get the explicit form of $\mathbf{A}^{+}$

$\mathbf{A}^{+}=\frac{1}{5 y^{2}+4 z^{2}}\left[\begin{array}{cc}2 y^{2}+2 z^{2} & 2 z \\ y z & 5 y \\ -y^{2} & 4 z\end{array}\right]$ 
Also, the control force $\mathbf{F}^{\mathbf{C}}$ can be explicitly found by Eq. (13). If there are multiple follower satellites, then we must use the original Udwadia-Kalaba equation (Eq. (10)) where the mass matrix $\mathbf{M}$ is given by $\mathbf{M}=$ $\operatorname{diag}\left\{m_{1}, m_{1}, m_{1}, m_{2}, m_{2}, m_{2}, \ldots, m_{N}, m_{N}, m_{N}\right\}$ and $N$ is the number of the follower satellites. The control force $\mathbf{F}^{\mathbf{C}}$ is then given by Eq. (8).

Thus, to find a solution to the formation-keeping problem, we use the Udwadia-Kalaba equation (Eq. (10) or (11)) in the Hill frame to get the explicit closed-form expression for the required control force. To this end, we need a, A, and b, which are given by Eqs. (24) and (30). What is left is only to determine a rotation matrix $\mathbf{R}$ in the expression for the unconstrained acceleration a given by Eq. (24) (recall, $\mathbf{S}=\mathbf{R}^{\mathbf{T}}$ ). Since the matrix $\mathbf{R}$ relates the ECI frame and the Hill frame, it depends on the trajectory that the leader is following. We will approach this problem in two equivalent ways. First, we will get a rotation matrix $\mathbf{R}$ and use our general approach in which the reference motion of the leader is prescribed as a function of time. Next, we will use a simpler case when the leader is in an unperturbed, Keplerian orbit. In this case, we can directly use the existing theory and do not have to obtain a rotation matrix $\mathbf{R}$. This simpler reference orbit is of course a special case of the general one, as will be shown later.

\subsection{General reference orbits}

Let us assume that the reference orbit is arbitrary, and the position and velocity vector of the leader are given by

$\mathbf{r}_{L}=\left[X_{L}(t), Y_{L}(t), Z_{L}(t)\right]^{\mathbf{T}}, \mathbf{v}_{L}=\left[\dot{X}_{L}(t), \dot{Y}_{L}(t), \dot{Z}_{L}(t)\right]^{\mathbf{T}}$.

This trajectory may be explicitly specified in time in advance, or it can be computed by integrating a suitable set of differential equations. It should be noted that these vectors are represented in the ECI frame. Then, the orbital angular momentum vector $\mathbf{h}_{L}$ is given by

$\mathbf{h}_{L}=\mathbf{r}_{L} \times \mathbf{v}_{L}$.

Now, we find the rotation matrix $\mathbf{R}$ that maps the ECI frame to the Hill frame, that is,

$\left[\begin{array}{c}x+\left\|\mathbf{r}_{L}\right\| \\ y \\ z\end{array}\right]=\mathbf{R}\left[\begin{array}{c}X_{L} \\ Y_{L} \\ Z_{L}\end{array}\right]$

To get the matrix $\mathbf{R}$, let us recall that we have three vector equations

$\left[\begin{array}{l}1 \\ 0 \\ 0\end{array}\right]=\mathbf{R} \hat{\mathbf{r}}_{L},\left[\begin{array}{l}0 \\ 1 \\ 0\end{array}\right]=\mathbf{R}\left(\hat{\mathbf{h}}_{L} \times \hat{\mathbf{r}}_{L}\right),\left[\begin{array}{l}0 \\ 0 \\ 1\end{array}\right]=\mathbf{R} \hat{\mathbf{h}}_{L}$,

where $\hat{\mathbf{r}}_{L} \triangleq \mathbf{r}_{L} /\left\|\mathbf{r}_{L}\right\|$ and $\hat{\mathbf{h}}_{L} \triangleq \mathbf{h}_{L} /\left\|\mathbf{h}_{L}\right\|$ (unit vectors). Then, we have

$\left[\begin{array}{lll}1 & 0 & 0 \\ 0 & 1 & 0 \\ 0 & 0 & 1\end{array}\right]=\mathbf{R}\left[\hat{\mathbf{r}}_{L}: \hat{\mathbf{h}}_{L} \times \hat{\mathbf{r}}_{L}: \hat{\mathbf{h}}_{L}\right]$
Since $\mathbf{R}$ is orthogonal, from Eq. (36) we obtain

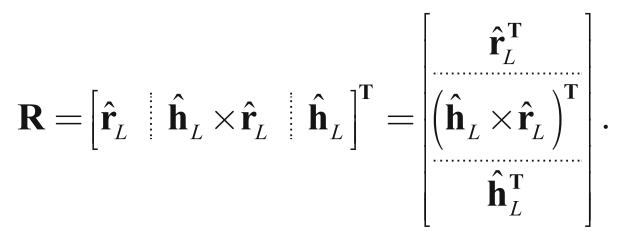

Each element of the matrices $\mathbf{R}, \dot{\mathbf{R}}$, and $\ddot{\mathbf{R}}$ is given in the Appendix. Having explicitly obtained the rotation matrix $\mathbf{R}$ given by Eq. (37), the uncontrolled acceleration a given by Eq. (24) can be found, and we can directly obtain the explicit control for precise formation-keeping given by using Eq. (8), and the equation of motion of the controlled system by using Eq. (10).

\subsection{Keplerian reference orbits}

Assuming Keplerian reference orbits (without perturbations), we can directly use the existing equations of relative motion expressed in the Hill frame [30]

$$
\begin{aligned}
& \ddot{x}=2 \dot{\theta}_{L} \dot{y}+\ddot{\theta}_{L} y+\dot{\theta}_{L}^{2} x-\frac{G M_{\oplus}\left(r_{L}+x\right)}{\left[\left(r_{L}+x\right)^{2}+y^{2}+z^{2}\right]^{3 / 2}}+\frac{G M_{\oplus}}{r_{L}^{2}}, \\
& \ddot{y}=-2 \dot{\theta}_{L} \dot{x}-\ddot{\theta}_{L} x+\dot{\theta}_{L}^{2} y-\frac{G M_{\oplus} y}{\left[\left(r_{L}+x\right)^{2}+y^{2}+z^{2}\right]^{3 / 2}},
\end{aligned}
$$

and

$\ddot{z}=-\frac{G M_{\oplus} z}{\left[\left(r_{L}+x\right)^{2}+y^{2}+z^{2}\right]^{3 / 2}}$,

where $\theta_{L}$ is the orbital rate of the leader and $r_{L}$ is the distance between the center of the Earth and the leader. In particular, $\theta_{L}$ can be treated as the true anomaly for elliptical reference orbits. Also, it is well-known that $\theta_{L}$ and $r_{L}$ are related by the following equations [30]

$r_{L}^{2} \dot{\theta}_{L}=h_{L}$

and

$r_{L}=\frac{h_{L}^{2} /\left(G M_{\oplus}\right)}{1+e_{L} \cos \theta_{L}}$

where $h_{L}$ is the magnitude of the constant orbital angular momentum vector of the leader and $e_{L}$ the eccentricity of the reference orbit. Eqs. (38)-(40) described in the Hill frame represent uncontrolled relative acceleration $\mathbf{a}$ of the follower with respect to the leader. Now we have all the quantities needed to place into the Udwadia-Kalaba equation: A, b, and a from Eqs. (30), (38)-(40). More explicitly, using Eq. (13) the required control force for a single follower can be explicitly obtained in a closed form as

$\mathbf{F}^{\mathbf{C}}=\frac{m}{5 y^{2}+4 z^{2}}\left[\begin{array}{c}-4\left(y^{2}+z^{2}\right) a_{x}-2 y z a_{y}+2 y^{2} a_{z}-2 z\left(\dot{y}^{2}+\dot{z}^{2}\right) \\ -2 y z a_{x}-5 y^{2} a_{y}-4 y z a_{z}-5 y\left(\dot{y}^{2}+\dot{z}^{2}\right) \\ 2 y^{2} a_{x}-4 y z a_{y}-\left(y^{2}+4 z^{2}\right) a_{z}-4 z\left(\dot{y}^{2}+\dot{z}^{2}\right)\end{array}\right]$,

where $a_{x}, a_{y}$, and $a_{z}$ are given by the right hand sides of Eqs. (38), (39), and (40), respectively. 


\section{Incorrect initial conditions}

The constraints must be satisfied at each instant of time during the maneuver including the initial time $(t=0)$. In practice, however, it is usually quite difficult to meet these constraints at the initial time because this requires inserting the follower(s) into orbit with the exact, required initial conditions. Hence, we need to modify our formulation; we do this by using Baumgarte's constraint stabilization method [31].

In general the constraint equations may be thought of as a set of $p$ constraints of the form

$\varphi_{i}(\mathbf{x}, \dot{\mathbf{x}}, t)=0, i=1,2, \ldots, p$,

where $\mathbf{x}$ is an $n$ by 1 generalized coordinate vector and $t$ represents time. For example, in this paper we have so far used two (that is, $p=2$ ) constraints given by Eqs. (25) and (26)

$\varphi_{1}=2 x-z=0$,

$\varphi_{2}=y^{2}+z^{2}-\rho^{2}=0$.

More compactly, we can write Eq. (44) as follows

$\boldsymbol{\Phi}=\left[\begin{array}{c}\varphi_{1} \\ \varphi_{2} \\ \vdots \\ \varphi_{p}\end{array}\right]=\mathbf{0}$.

In this paper, Eq. (47) becomes

$\mathbf{\Phi}(t)=\left[\begin{array}{c}2 x(t)-z(t) \\ y(t)^{2}+z(t)^{2}-\rho^{2}\end{array}\right]=\mathbf{0}$.

By differentiating the above equation with respect to time appropriately, we get the general constraint equations mentioned earlier

$\mathbf{A}(\mathbf{x}, \dot{\mathbf{x}}, t) \ddot{\mathbf{x}}(t)=\mathbf{b}(\mathbf{x}, \dot{\mathbf{x}}, t)$.

In this paper, the constraint equation in the form of Eq. (6) is given by Eq. (29).

Now, we consider general initial conditions that do not satisfy the given constraints, which means $\boldsymbol{\Phi}(0) \neq \mathbf{0}$ at the initial time. We modify the constraint Eq. (47) to [31]

$\ddot{\boldsymbol{\Phi}}+\boldsymbol{\alpha} \dot{\boldsymbol{\Phi}}+\boldsymbol{\beta} \boldsymbol{\Phi}=\mathbf{0}$,

where

$\boldsymbol{\alpha}=\operatorname{diag}\left\{\alpha_{1}, \alpha_{2}, \ldots, \alpha_{p}\right\}, \boldsymbol{\beta}=\operatorname{diag}\left\{\beta_{1}, \beta_{2}, \ldots, \beta_{p}\right\}$.

It is well-known that if each $\alpha_{i}, \beta_{i}>0, i=1,2, \ldots, p, \boldsymbol{\Phi}$ approaches to zero asymptotically. Thus, from Eq. (49), we get a more general constraint equation, which nonetheless retains the form of Eq. (6).

In this paper so far we have, $p=2$, so both $\alpha$ and $\boldsymbol{\beta}$ are $2 \times 2$ diagonal matrices and the 2 by 1 matrix $\Phi$ is given by Eq. (48). Then, Eq. (49) yields the following constraint equation

$$
\left[\begin{array}{ccc}
2 & 0 & -1 \\
0 & y & z
\end{array}\right]\left[\begin{array}{c}
\ddot{x} \\
\ddot{y} \\
\ddot{z}
\end{array}\right]=\left[\begin{array}{c}
-\alpha_{1}(2 \dot{x}-\dot{z})-\beta_{1}(2 x-z) \\
-\dot{y}^{2}-\dot{z}^{2}-\alpha_{2}(y \dot{y}+z \dot{z})-\frac{\beta_{2}}{2}\left(y^{2}+z^{2}-\rho^{2}\right)
\end{array}\right],
$$

which is of the form of $\mathbf{A} \ddot{\mathbf{x}}=\mathbf{b}$ or Eq. (6). If the initial conditions do not meet the constraints, we must utilize Eq. (49) instead of Eq. (47) and use these new quantities A and $\mathbf{b}$ in Eqs. (8) and (10) respectively to get the explicit closed-form expressions for the required control force and the explicit equation of motion of the controlled system.

\section{Numerical example}

In this section, the explicit analytic results given in Sections 4 and 5 are verified by numerical simulations. We attempt to find the required control force that will keep the relative configuration in a circle and a straight line when projected onto the $y z$ and $x z$ planes in the Hill frame, respectively. In this section we present two examples.

In Example 1, there is only one follower satellite, the leader's reference orbit is elliptical, and the initial conditions satisfy the given constraints. In Example 2, we have two follower satellites, the leader has a more general motion described by a spiral, and the initial conditions for the followers do not meet the constraints, i.e., the followers are not correctly inserted into their desired orbits initially. In Example 1, since the leader's reference orbit is elliptical, we use the two methods explained in the previous section and show that they yield very close numerical results. We note that while the leader's reference orbit in Example 2 is chosen to be a spiral, the same control strategy can be easily applied to any other prescribed reference orbits.

The numerical integration throughout this paper is done in the Matlab environment, using a variable time step integrator with a relative error tolerance of $10^{-12}$ and an absolute error tolerance of $10^{-20}$.

\subsection{Example 1: one follower, elliptical reference orbit, correct initial conditions}

Let us assume that the mass of the follower is $1000 \mathrm{~kg}$, the semi-major axis $\left(a_{L}\right)$ and the eccentricity $\left(e_{L}\right)$ of the leader's orbit (or reference orbit) are $7.0 \times 10^{6} \mathrm{~m}$ and 0.1 , respectively. We desire the follower to maintain a projected circular orbit (PCO) with $\rho=50 \mathrm{~km}$, and the initial conditions are given by

$$
\begin{aligned}
& x(0)=1.30672 \times 10^{4} \mathrm{~m}, \\
& y(0)=4.26262 \times 10^{4} \mathrm{~m}, \\
& z(0)=2.61344 \times 10^{4} \mathrm{~m}, \\
& \dot{x}(0)=22.9784 \mathrm{~m} / \mathrm{s}, \\
& \dot{y}(0)=-28.1764 \mathrm{~m} / \mathrm{s}, \\
& \dot{z}(0)=45.9568 \mathrm{~m} / \mathrm{s} .
\end{aligned}
$$

It should be noted that the initial conditions given in Eq. (52) satisfy the two constraints (Eqs. (25) and (26)).

First, we find a solution by using the method given by Section 4.1. At $t=0$, let us assume that the leader is at the perigee which is located at the ascending node. Also, the longitude of the ascending node $\left(\Omega_{L}\right)$ and the inclination $\left(i_{L}\right)$ of the leader are given by $30^{\circ}$ and $80^{\circ}$, respectively. The initial position and speed of the leader in 
the ECI frame can then be calculated by the following equations [29]

$r_{L}(0)=a_{L}\left(1-e_{L}\right)=6.3 \times 10^{6} \mathrm{~m}$,

$v_{L}(0)=\sqrt{\frac{2 G M_{\oplus}}{r_{L}(0)}-\frac{G M_{\oplus}}{a_{L}}}=8.34346 \times 10^{3} \mathrm{~m} / \mathrm{s}$.

Hence, the initial conditions for the leader in the ECI frame are:

$X_{L}(0)=r_{L}(0) \cos \left(\Omega_{L}\right)=5.45596 \times 10^{6} \mathrm{~m}$,

$Y_{L}(0)=r_{L}(0) \sin \left(\Omega_{L}\right)=3.15 \times 10^{6} \mathrm{~m}$,

$Z_{L}(0)=0 \mathrm{~m}$,

$\dot{X}_{L}(0)=-v_{L}(0) \cos \left(i_{L}\right) \sin \left(\Omega_{L}\right)=-7.24414 \times 10^{2} \mathrm{~m} / \mathrm{s}$,

$\dot{Y}_{L}(0)=v_{L}(0) \cos \left(i_{L}\right) \cos \left(\Omega_{L}\right)=1.25472 \times 10^{3} \mathrm{~m} / \mathrm{s}$,

$\dot{Z}_{L}(0)=v_{L}(0) \sin \left(i_{L}\right)=8.21671 \times 10^{3} \mathrm{~m} / \mathrm{s}$.

With the initial conditions given in Eq. (54), we use the given motion of the leader to obtain the rotation matrix $\mathbf{R}$ given by Eq. (37) and thence (see, Appendix) the uncontrolled acceleration a given by Eq. (24). We can use Eqs. (11) and (13) to get the controlled motion and the explicit control force to be applied to the follower. The quantities a, A, $\mathbf{A}^{+}$, and $\mathbf{b}$ are given by Eqs. (24), (30), and (31). The duration of time used for the numerical integration is three times the orbital period, $P_{L}$, of the leader which is given by

$P_{L}=\frac{2 \pi}{\bar{n}_{L}}=5.82783 \times 10^{3} \mathrm{~s}=1.61884 \mathrm{~h}$,

where $\bar{n}_{L}$ is the mean motion of the leader obtained from Kepler's third law as

$\bar{n}_{L}=\sqrt{\frac{G M_{\oplus}}{a_{L}^{3}}}=1.07814 \times 10^{-3} \mathrm{rad} / \mathrm{s}$.

Figs. 3 and 4 represent the orbits of the follower projected on the $x z$-plane in the Hill frame without and with control, respectively. The scale is normalized by $\rho$. Without control, the follower shows complex motion, whereas the follower moves exactly along the constraint line $(2 x=z)$ with the control force. Fig. 5 depicts the orbit of the follower projected on the $y z$-plane in the Hill frame without control. The motion of the follower satellite is unbounded as seen from its leftwards motion in the $y$ direction. In Fig. 6, the controlled motion of the follower is reported. The trajectory is being maintained very well with the relative distance of $\rho=50 \mathrm{~km}$.

In Fig. 7 we show the explicitly obtained control force per unit mass of the follower to maintain the desired formation and its total magnitude. The force components are described in the Hill frame and calculated using Eq. (13). Time is normalized to the period of the leader $\left(P_{L}\right)$.

Figs. 8-11 represent numerical errors in the satisfaction of the constraints described by Eqs. (25)(28), which are denoted by $e_{1}(t)=2 x-z, e_{2}(t)=\sqrt{y^{2}+z^{2}}$ $-\rho, e_{3}(t)=2 \dot{x}-\dot{z}$, and $e_{4}(t)=y \dot{y}+z \dot{z}$. We see that these errors have orders of magnitude that are commensurate

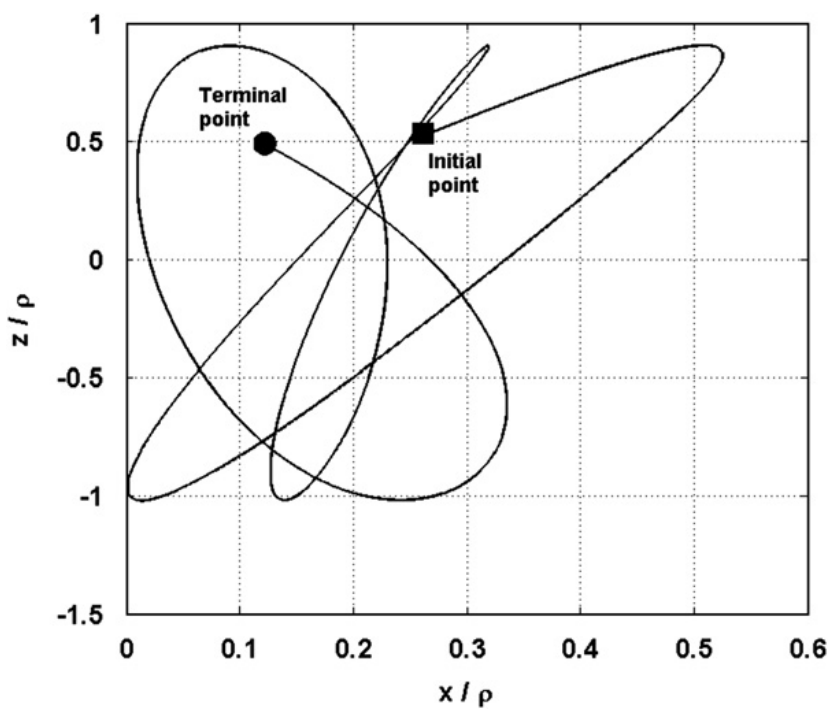

Fig. 3. Uncontrolled motion in the $x z$-plane.

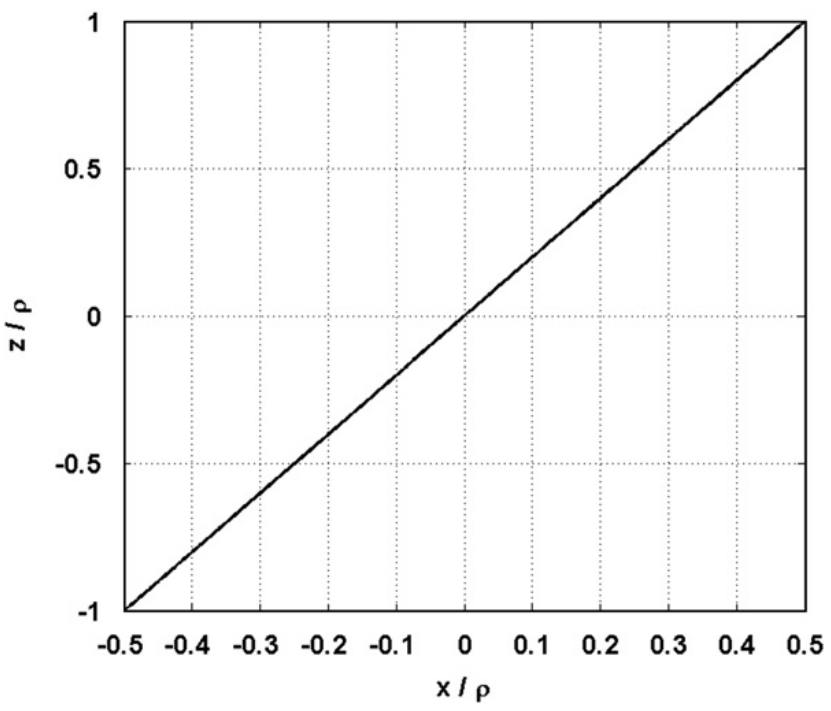

Fig. 4. Controlled motion in the $x z$-plane.

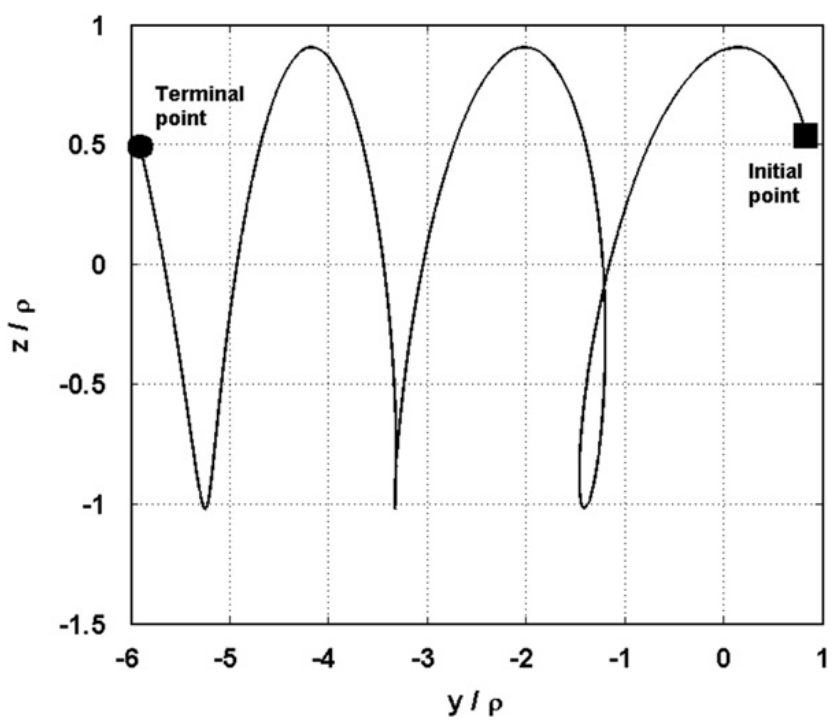

Fig. 5. Uncontrolled motion in the $y z$-plane. 
with the relative tolerance used in the numerical integration of the equations of motion.

Next, we approach the same problem via the method given by Section 4.2. We use the same initial conditions for the follower (Eq. (52)) to get the solution. The quantities $\mathbf{a}, \mathbf{A}, \mathbf{A}^{+}$, and $\mathbf{b}$ are explicitly given by Eqs. (24), (30), and (31), and the required control forces can be directly obtained by Eq. (43). As seen in Eq. (42), however, $r_{L}$ is an explicit function of the true anomaly $\left(\theta_{L}\right)$ of the leader, not time, so in order to express $r_{L}$ as a function of time, Kepler's equation [30] must be solved. In this paper, we used the well-known Newton-Raphson numerical method [30] to do this. As expected, the results obtained are almost the same as the previous ones. In Fig. 12, the difference of the required forces and their total magnitude is represented. They are described in the Hill frame and we can see that the difference is very small when recalling the original thrust magnitude depicted in Fig. 7. Fig. 13 depicts the difference between each component of the relative position vector in the Hill frame obtained using the general method (Section 4.1) and the special method

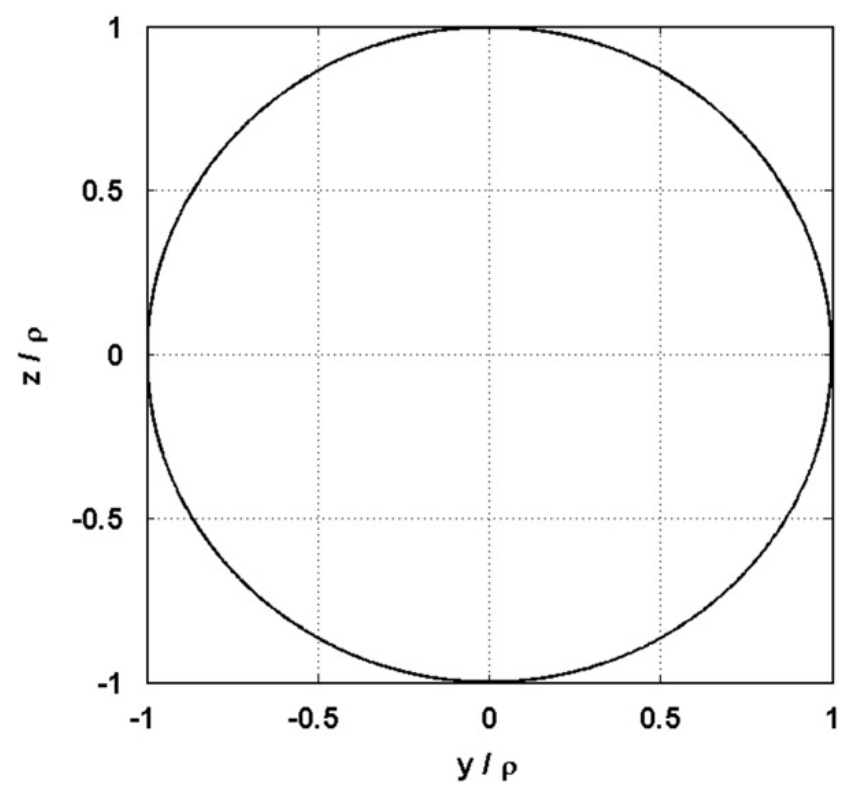

Fig. 6. Controlled motion in the $y z$-plane.
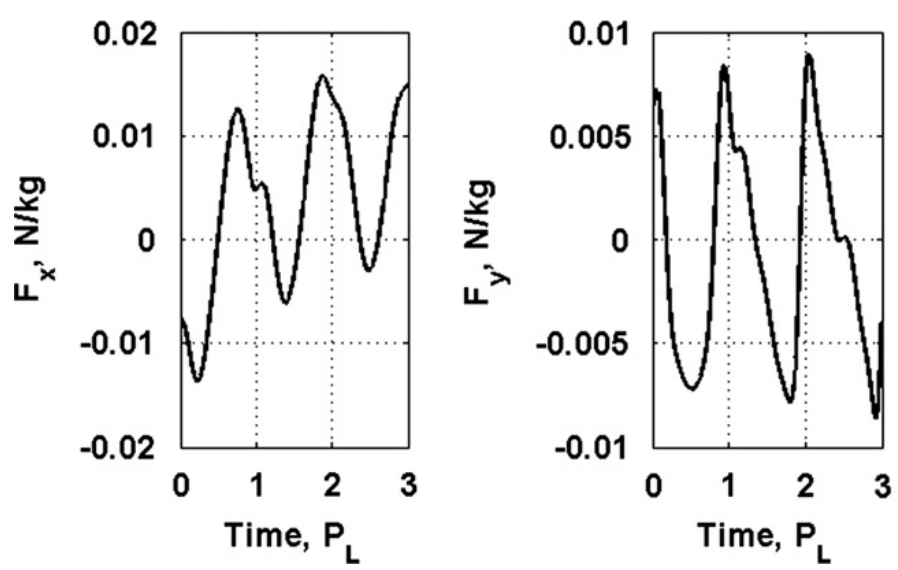

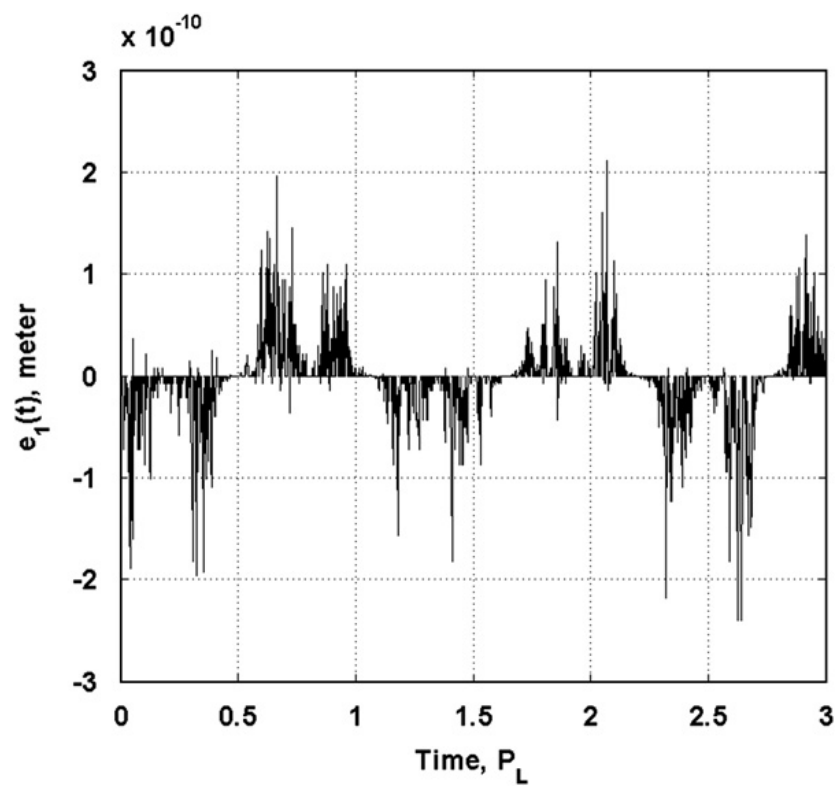

Fig. 8. Displacement error for the first constraint.

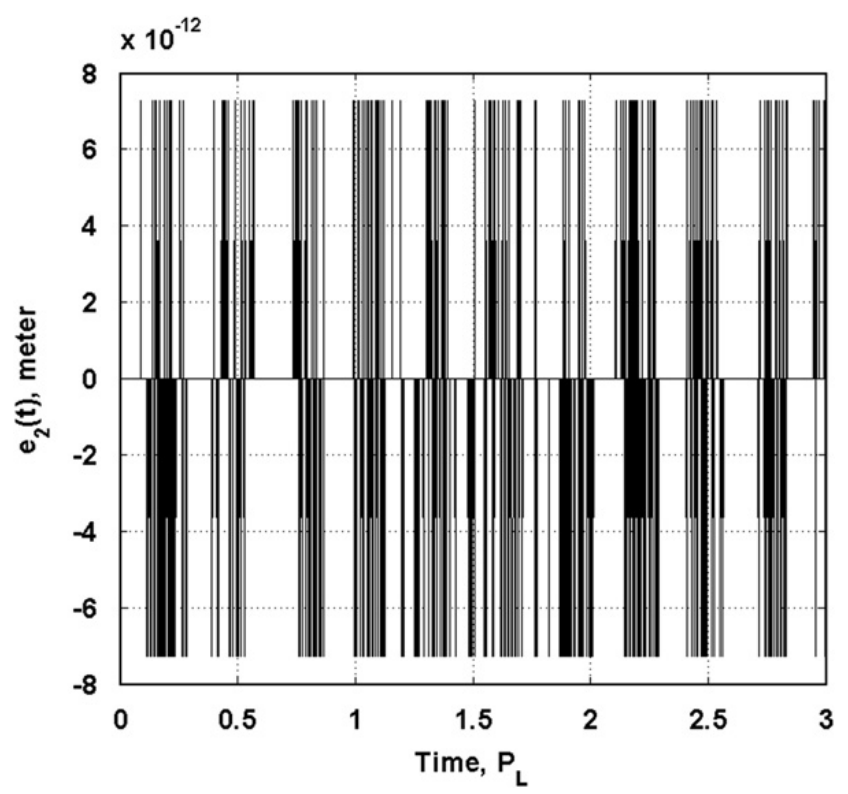

Fig. 9. Displacement error for the second constraint.
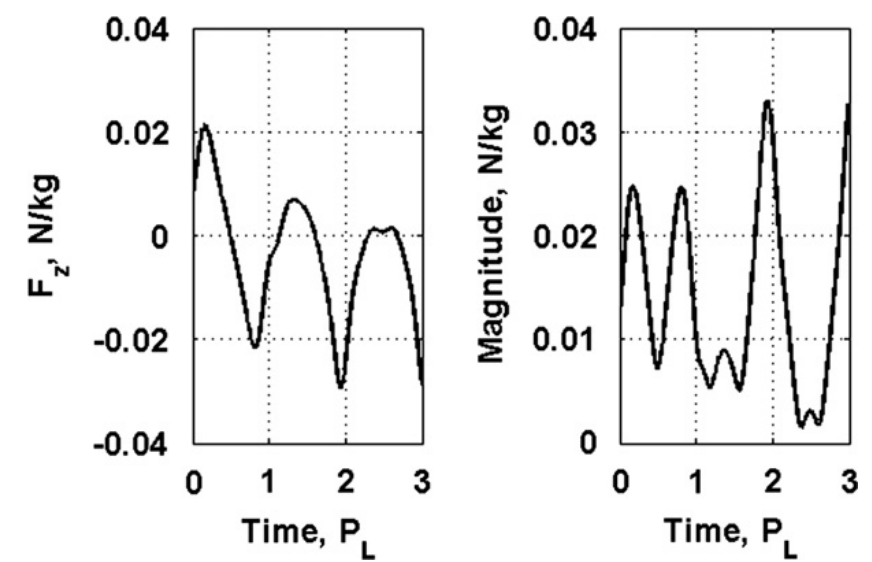

Fig. 7. Components of the required control force in the Hill frame and its magnitude. 


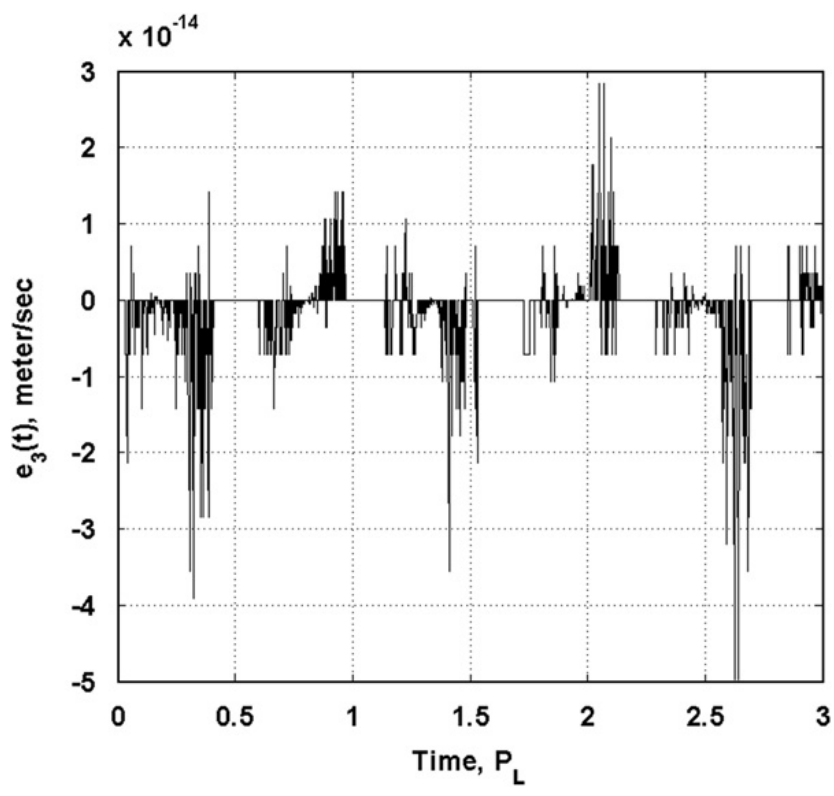

Fig. 10. Velocity error for the first constraint.

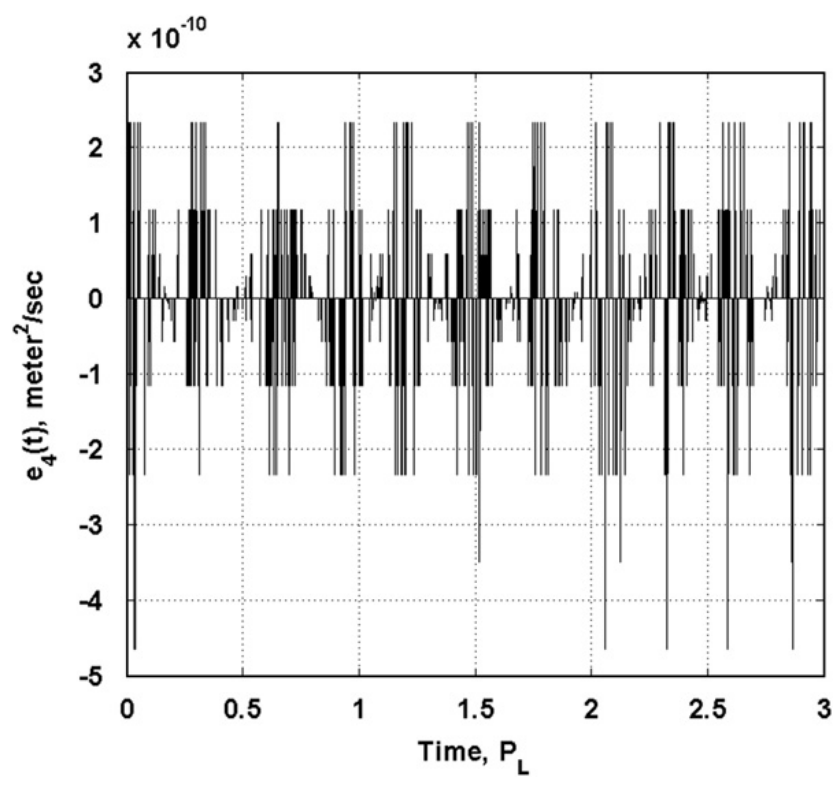

Fig. 11. Velocity error for the second constraint.
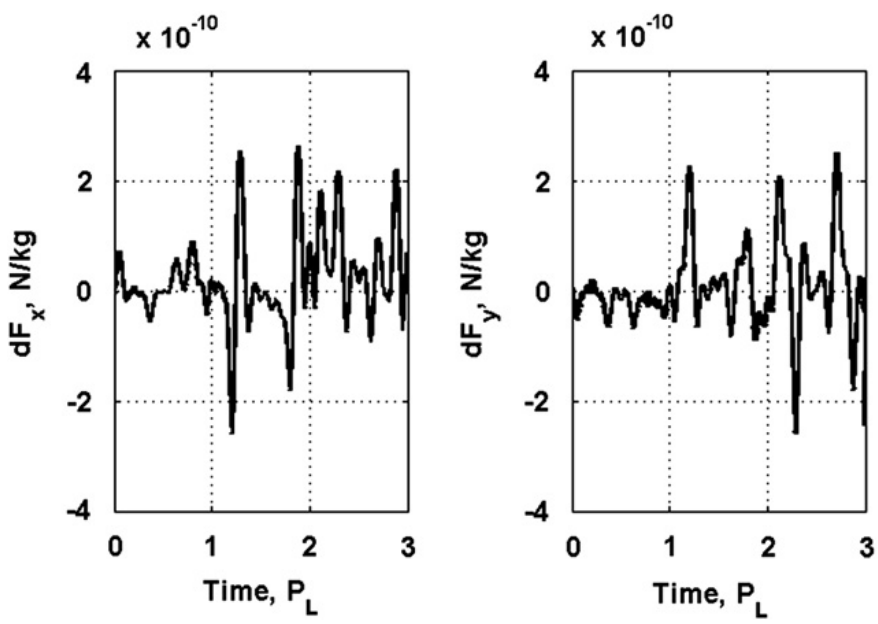

(Section 4.2). The difference is of the order of $10^{-4} \mathrm{~m}$, which is very small when compared with the relative orbit size $\rho=50 \mathrm{~km}$. This verifies that the general method explained in Section 4.1 includes the special case introduced in Section 4.2.

\subsection{Example 2: two followers, spiral reference orbit, incorrect initial conditions}

This example is more general than the previous one. In this case, there are two followers, and the reference orbit is a spiral, defined as

$X_{L}=a_{L} \cos \left(\bar{n}_{L} t\right), Y_{L}=a_{L} \sin \left(\bar{n}_{L} t\right), Z_{L}=k_{L} t$,

where $a_{L}=7.0 \times 10^{6} \mathrm{~m}, \bar{n}_{L}=1.07814 \times 10^{-3} \mathrm{rad} / \mathrm{s}$ is the same mean motion given by Eq. (56), and $k_{L}=100 \mathrm{~m} / \mathrm{s}$. The leader's trajectory in the ECI frame in 3-dimensions is shown in Fig. 14. Also, the initial conditions for the first follower (named Follower 1) whose mass is $1000 \mathrm{~kg}$ are given by

$x_{1}(0)=1.76777 \times 10^{4} \mathrm{~m}, \quad y_{1}(0)=3.63553 \times 10^{4} \mathrm{~m}$,

$z_{1}(0)=3.03553 \times 10^{4} \mathrm{~m}$,

$\dot{x}_{1}(0)=14.2942 \mathrm{~m} / \mathrm{s}$,

$\dot{y}_{1}(0)=-28.5884 \mathrm{~m} / \mathrm{s}, \quad \dot{z}_{1}(0)=28.5884 \mathrm{~m} / \mathrm{s}$,

where the subscript "1" denotes Follower 1, and the initial conditions for the second follower (named Follower 2) whose mass is $800 \mathrm{~kg}$ are

$x_{2}(0)=-1.78544 \times 10^{4} \mathrm{~m}$,

$y_{2}(0)=-3.67189 \times 10^{4} \mathrm{~m}, \quad z_{2}(0)=-3.06589 \times 10^{4} \mathrm{~m}$,

$\dot{x}_{2}(0)=-14.2942 \mathrm{~m} / \mathrm{s}$,

$\dot{y}_{2}(0)=28.5884 \mathrm{~m} / \mathrm{s}, \quad \dot{z}_{2}(0)=-28.5884 \mathrm{~m} / \mathrm{s}$,

where the subscript "2" denotes Follower 2. Here, in order to stress the strength of this paper we set more complex and specific constraints instead of the ones in Eqs. (25) and (26) so that the followers execute a circular orbit of radius $\rho=50 \mathrm{~km}$ around the leader with a prescribed angular frequency $\omega$ in the Hill frame. In addition, we add one more constraint: as seen in the Hill frame at all times, the two followers must be located diametrically opposite
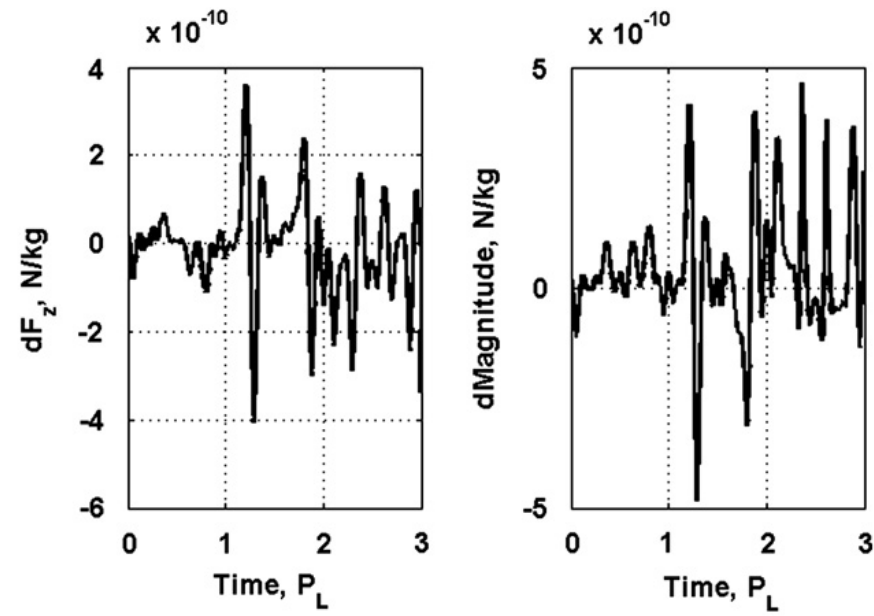

Fig. 12. Difference of the required forces and their total magnitude using the general reference orbit formulation given in Section 4.1 and the elliptical reference orbit formulation given in Section 4.2. 

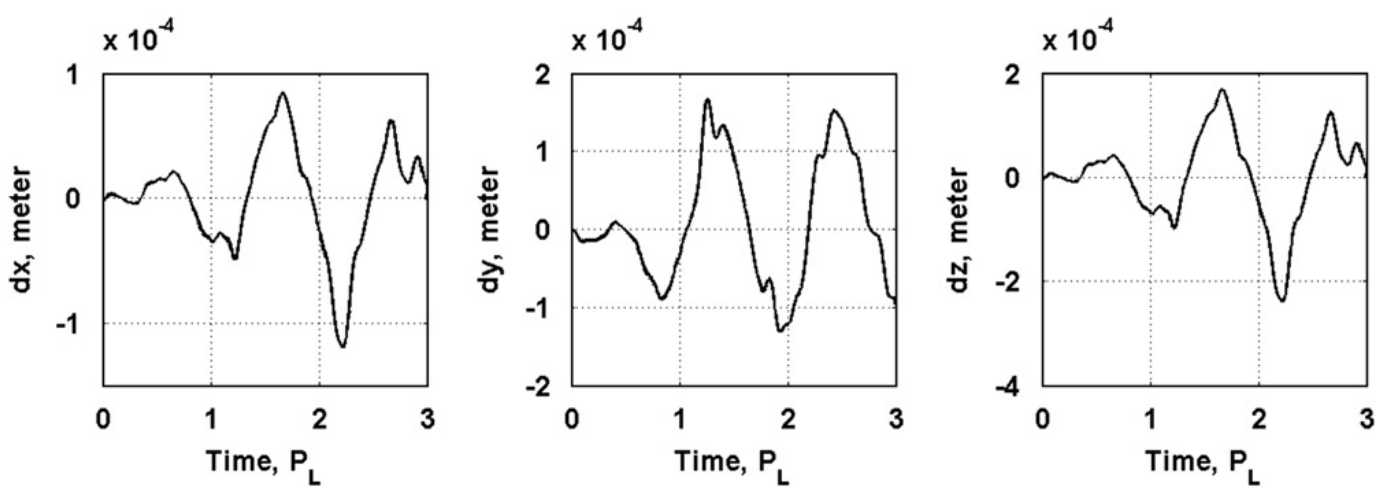

Fig. 13. Difference between each component of the relative position vector in the Hill frame when using the general formulation and that particularized to Keplerian reference orbits.

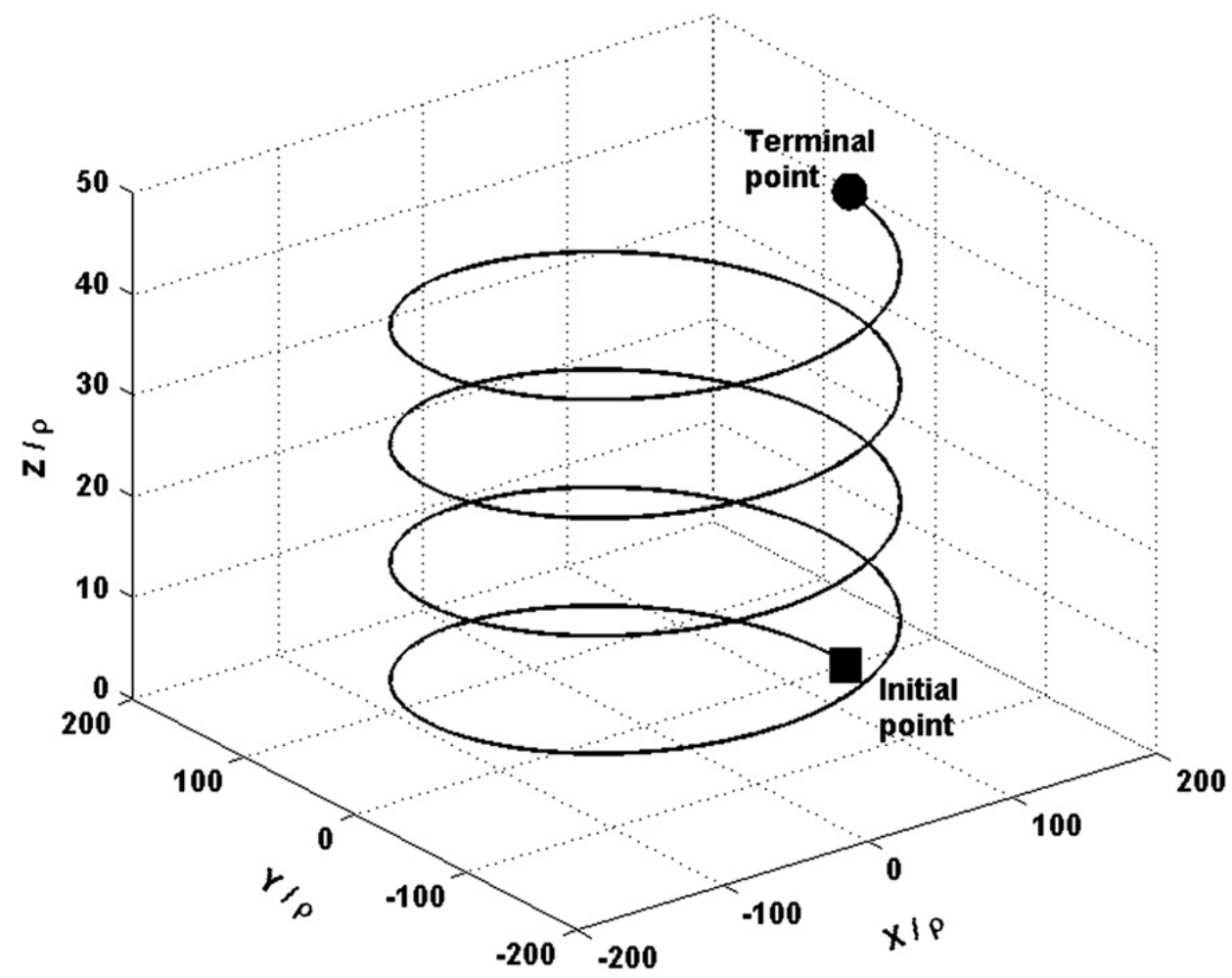

Fig. 14. Leader's trajectory in the ECI frame in 3-D.

each other in their orbits around the leader; that is, the leader is located at the center of the line joining the two followers. There are thus the following six constraints in total:

$2 x_{1}=z_{1}$,

$y_{1}=\rho \cos (\omega t+\varphi)$,

$z_{1}=\rho \sin (\omega t+\varphi)$

$2 x_{2}=z_{2}$,

$y_{2}=-\rho \cos (\omega t+\varphi)$,

$z_{2}=-\rho \sin (\omega t+\varphi)$.

Although $\omega$ and $\varphi$ can have any values, for illustrative purposes we use $\omega=0.75 \bar{n}_{L}$ and $\varphi=\pi / 4$, where $\bar{n}_{L}$ is given by Eq. (56). Then, the followers revolve around the leader three times in the Hill frame while the leader's orbit makes four revolutions when projected onto the $X Y$-plane in the ECI frame. Here, it should be noted that the initial conditions for Followers 1 and 2 do not meet the six constraints given by Eq. (60).

We have the following constraint matrix $\boldsymbol{\Phi}$ in the form of Eq. (47)

$$
\mathbf{\Phi}=\left[\begin{array}{c}
2 x_{1}-z_{1} \\
y_{1}-\rho \cos (\omega t+\varphi) \\
z_{1}-\rho \sin (\omega t+\varphi) \\
2 x_{2}-z_{2} \\
y_{2}+\rho \cos (\omega t+\varphi) \\
z_{2}+\rho \sin (\omega t+\varphi)
\end{array}\right]=\mathbf{0} .
$$

However, since the initial conditions do not meet the constraints, we must use Eq. (49) instead of Eq. (47) to get A and b. Inserting Eq. (61) into (49), we get the following 
constraint equation, in the form of $\mathbf{A} \ddot{\mathbf{x}}=\mathbf{b}$, given by

$$
\begin{gathered}
{\left[\begin{array}{cccccc}
2 & 0 & -1 & 0 & 0 & 0 \\
0 & 1 & 0 & 0 & 0 & 0 \\
0 & 0 & 1 & 0 & 0 & 0 \\
0 & 0 & 0 & 2 & 0 & -1 \\
0 & 0 & 0 & 0 & 1 & 0 \\
0 & 0 & 0 & 0 & 0 & 1
\end{array}\right]\left[\begin{array}{l}
\ddot{x}_{1} \\
\ddot{y}_{1} \\
\ddot{z}_{1} \\
\ddot{x}_{2} \\
\ddot{y}_{2} \\
\ddot{z}_{2}
\end{array}\right]} \\
=\left[\begin{array}{c}
-\alpha_{1}\left(2 \dot{x}_{1}-\dot{z}_{1}\right)-\beta_{1}\left(2 x_{1}-z_{1}\right) \\
-\omega^{2} \rho \cos (\omega t+\varphi)-\alpha_{2}\left(\dot{y}_{1}+\omega \rho \sin (\omega t+\varphi)\right)-\beta_{2}\left(y_{1}-\rho \cos (\omega t+\varphi)\right) \\
-\omega^{2} \rho \sin (\omega t+\varphi)-\alpha_{3}\left(\dot{z}_{1}-\omega \rho \cos (\omega t+\varphi)\right)-\beta_{3}\left(z_{1}-\rho \sin (\omega t+\varphi)\right) \\
\omega^{2} \rho \cos (\omega t+\varphi)-\alpha_{5}\left(\dot{y}_{2}-\omega \rho \sin (\omega t+\varphi)\right)-\beta_{5}\left(y_{2}+\rho \cos (\omega t+\varphi)\right) \\
\omega^{2} \rho \sin (\omega t+\varphi)-\alpha_{6}\left(\dot{z}_{2}+\omega \rho \cos (\omega t+\varphi)\right)-\beta_{6}\left(z_{2}+\rho \sin (\omega t+\varphi)\right)
\end{array}\right]
\end{gathered}
$$

From Eq. (62), the 6 by 6 matrix $\mathbf{A}$ and the 6 by 1 vector $\mathbf{b}$ are both obvious.

For simplicity, we choose $\alpha_{i}=0.002, \quad \beta_{i}=0.001$, $i=1,2, \ldots, 6$. There are two followers, so we use Eqs. (8) and (10) to get the explicit control forces and the controlled motion of the two followers. The spiral orbit specified by Eq. (57) is no longer a Keplerian orbit, so we must use the general method explained in Section 4.1, and a, A, and $\mathbf{b}$ are given by Eqs. (24) and (62). In this case, the matrices $\mathbf{A}$ and $\mathbf{A M}^{-1 / 2}$ are both 6 by 6 and nonsingular, so the Moore-Penrose generalized inverse of $\mathbf{A M} \mathbf{M}^{-1 / 2}$ is just its regular inverse. Using the fact that $\left(\mathbf{A} \mathbf{M}^{-1 / 2}\right)^{-1}=\mathbf{M}^{1 / 2} \mathbf{A}^{-1}$, Eqs. (8) and (10) become very simple:

$\mathbf{F}^{\mathbf{C}}=\mathbf{M}\left(\mathbf{A}^{-1} \mathbf{b}-\mathbf{a}\right)$,

and

$\ddot{\mathbf{x}}=\mathbf{A}^{-1} \mathbf{b}$

where

$\mathbf{A}^{-1}=\left[\begin{array}{cccccc}0.5 & 0 & 0.5 & 0 & 0 & 0 \\ 0 & 1 & 0 & 0 & 0 & 0 \\ 0 & 0 & 1 & 0 & 0 & 0 \\ 0 & 0 & 0 & 0.5 & 0 & 0.5 \\ 0 & 0 & 0 & 0 & 1 & 0 \\ 0 & 0 & 0 & 0 & 0 & 1\end{array}\right]$.

The duration of time over which the numerical integration is carried out equals four times the orbital period of the leader in the $X Y$ inertial frame, that is, $4 P_{L}$ where $P_{L}$ is given by Eq. (55).

Figs. 15 and 16 show the controlled and uncontrolled orbits projected onto the $x z$ and $y z$ planes for Followers 1 and 2 , respectively. As before, the scale is normalized to $\rho$. As expected, the uncontrolled motion is unbounded especially in the $z$ direction. For the controlled motion, the orbits do not satisfy the constraints initially, but they merge onto the constraint circle (and onto the line) progressively with time.
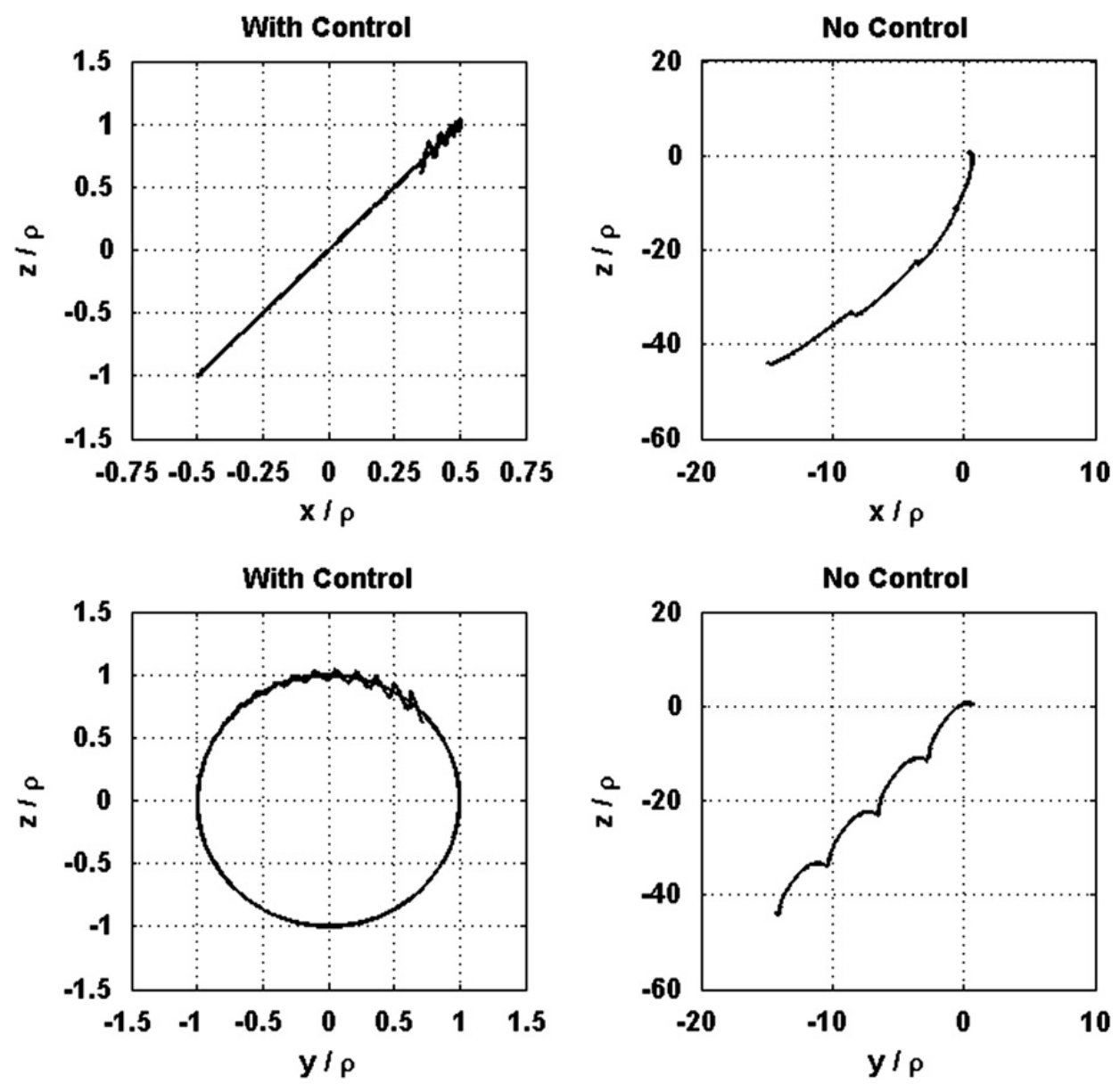

Fig. 15. Controlled and uncontrolled trajectories projected onto the $x z$ and $y z$ planes for Follower 1. 

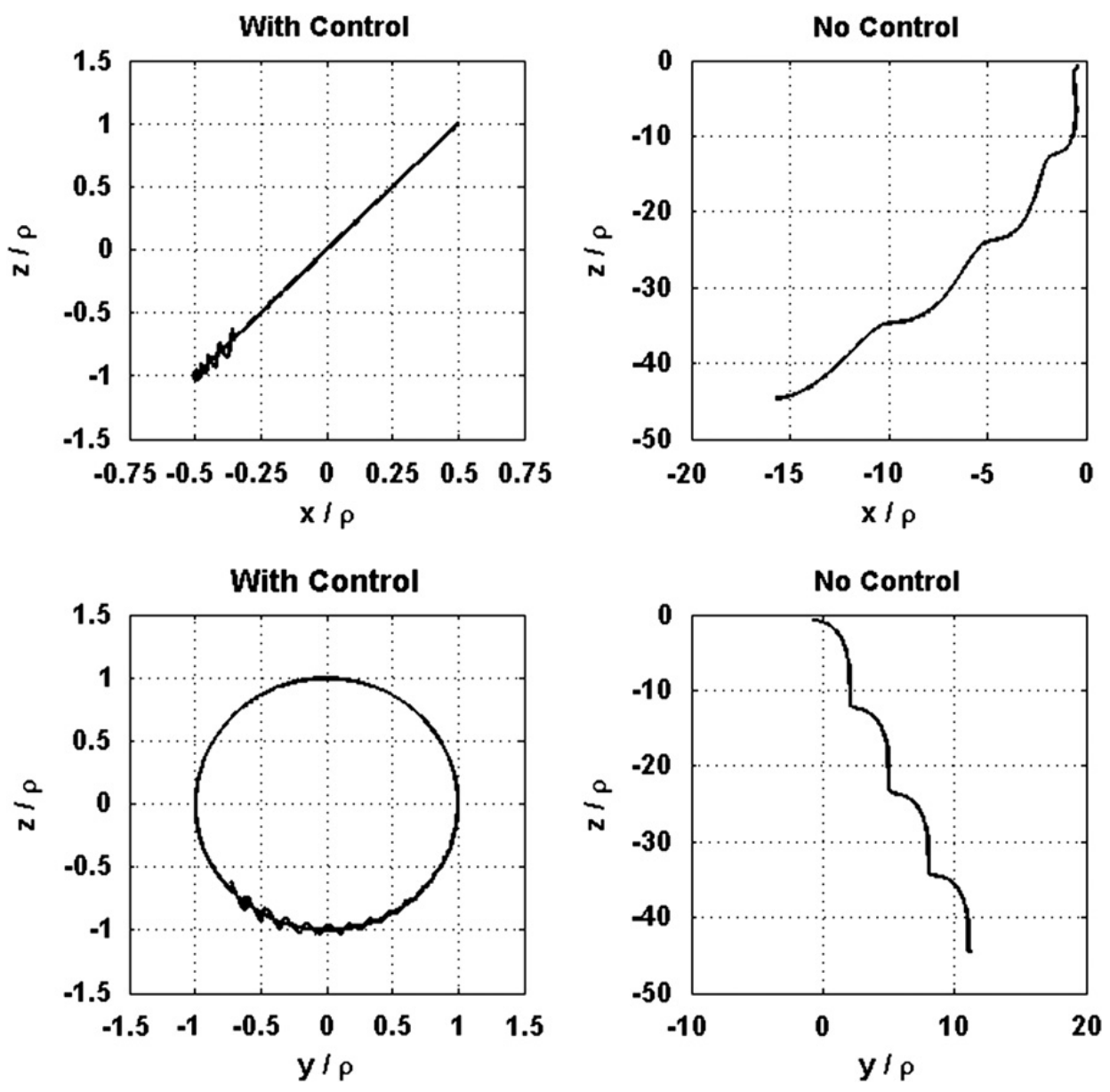

Fig. 16. Controlled and uncontrolled trajectories projected onto the $x z$ and $y z$ planes for Follower 2 .

Fig. 17 shows the controlled motion of the two followers in each axis. The solid lines represent Follower 1 's controlled trajectory and the dashed lines represent Follower 2's controlled trajectory. As seen they are diametrically opposite each other in the $y z$-plane, and the plots for $x(t)$ and $z(t)$ have the same shape except for a scaling by two due to the constraints $2 x_{1}=z_{1}$ and $2 x_{2}=z_{2}$. Also, they are sinusoidal with three oscillations (as seen in the Hill frame) for four revolutions $\left(4 P_{L}\right)$ of the leader in the $X Y$-plane because the angular frequency $\omega$ is set as $\omega=0.75 \bar{n}_{L}$.

Fig. 18 shows the numerical errors in the satisfaction of the constraints specified in Eq. (60). Denoting $e_{1}(t)=2 x_{1}-z_{1}, \quad e_{2}(t)=y_{1}-\rho \cos (\omega t+\varphi), \quad e_{3}(t)=z_{1}-\rho$ $\sin (\omega t+\varphi), e_{4}(t)=2 x_{2}-z_{2}, \quad e_{5}(t)=y_{2}+\rho \cos (\omega t+\varphi)$, and $e_{6}(t)=z_{2}+\rho \sin (\omega t+\varphi)$, we see that initially these errors are very large since the follower satellites have not been inserted into orbit with the correct initial conditions that satisfy the desired constraints. More specifically, these initial errors $e_{i}(0), i=1,2,3,4,5,6$ are of the order of $10^{3} \mathrm{~m}$ in all. However, they converge to zero as time progresses, and at the final time (four revolutions of the leader in the $X Y$-frame), the final errors $e_{i}\left(4 P_{L}\right)$, $i=1,2,3,4,5,6$ are of the order of $10^{-8}, 10^{-9}, 10^{-8}$, $10^{-8}, 10^{-9}$, and $10^{-8} \mathrm{~m}$, respectively.

In Figs. 19 and 20, the required control forces (per unit mass) to maintain the desired formation and their total magnitude are shown. They are described in the Hill frame and calculated using Eq. (8) (or Eq. (63)). Fig. 19 shows the control forces and their total magnitude for both followers over the duration 0 to $1 P_{L}$ secs. As seen, relatively large control forces are brought into play over this initial time duration to try and eliminate the large initial insertion errors in the orbits of the two followers. The maximum magnitudes of these control forces are $5.109990 \mathrm{~N} / \mathrm{kg}$ for Follower 1 and $4.90475 \mathrm{~N} / \mathrm{kg}$ for Follower 2. While such large force may be hard to be applied to real missions, it shows, however, that the general method developed in this paper can easily apply to general and more complex examples. One can, of course, reduce these magnitudes by choosing smaller $\alpha_{i}^{\prime}$ 's and $\beta_{i}$ 's than those used here; but this would come at the expense of a longer time duration needed to get the followers to their required PCO orbits. In Fig. 20, the control forces and their total magnitude for both followers over the duration $1 P_{L}$ to $4 P_{L}$ secs. are shown. It is noted that the magnitude of thrust in the $z$ direction dominates the total magnitude for both followers. Also, the magnitude of the total thrust is gradually increasing for each follower. Thus, as the leader gets more distant from the Earth, more thrust is needed to control the followers' motion. As seen in Eq. (63), the control force $\mathbf{F}^{\mathbf{C}}$ is composed of two components: $\mathbf{M A}^{-1} \mathbf{b}$ and Ma. However, the matrices 

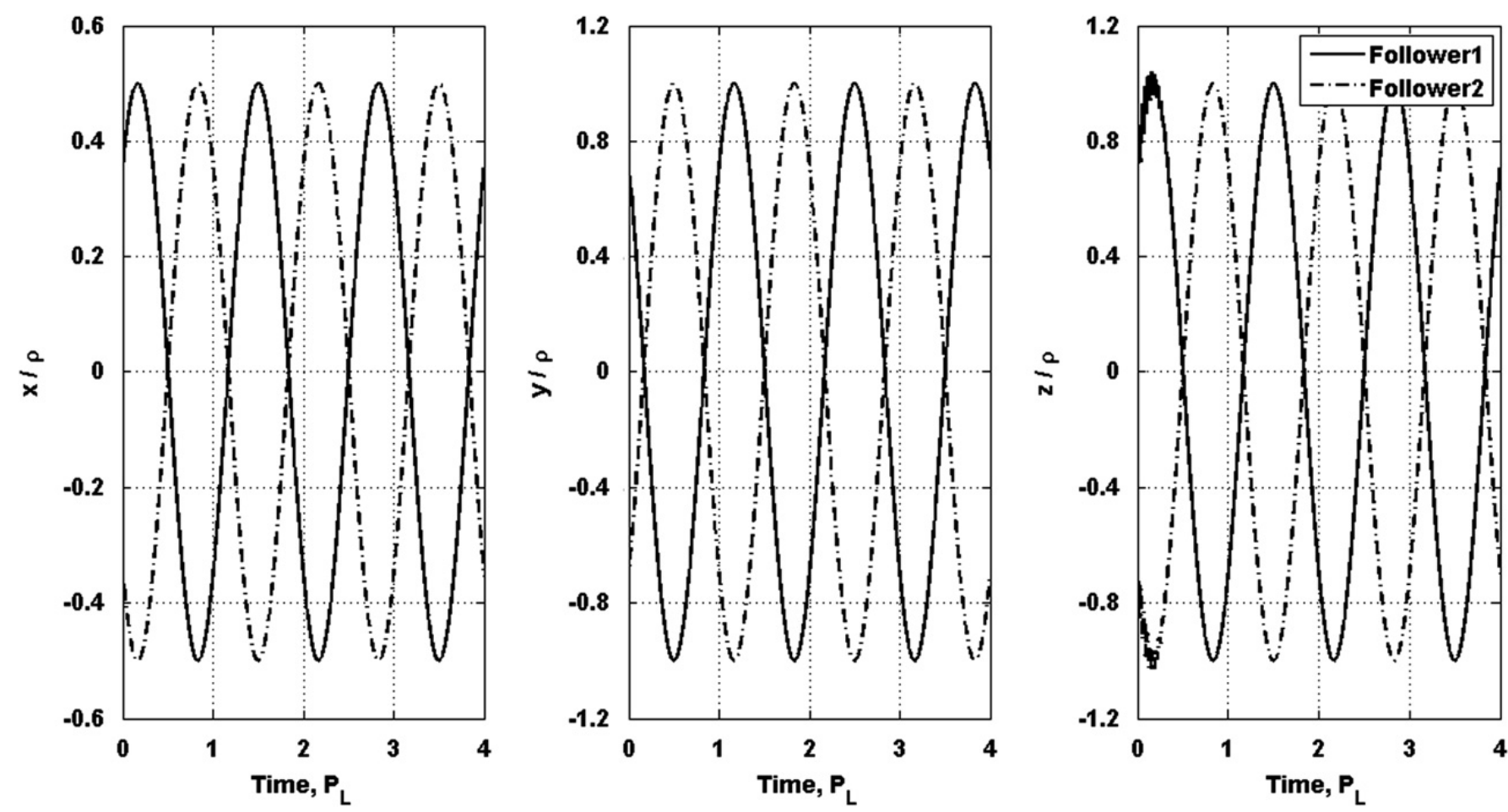

Fig. 17. Orbits in the Hill frame of the two followers.
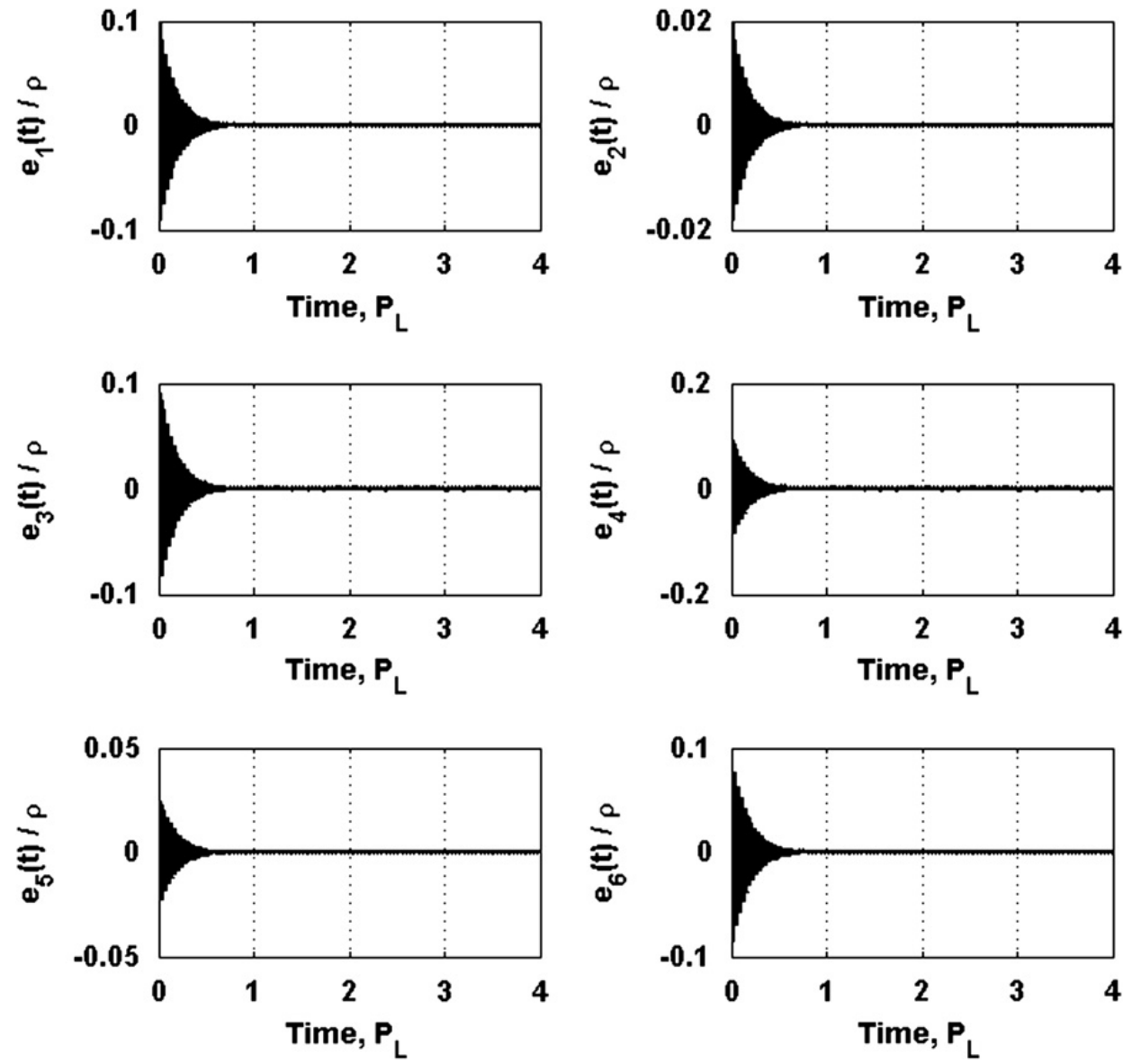

Fig. 18. Numerical errors in satisfaction of the six constraints. Note the values of $e_{i}(0), i=1,2,3,4,5,6$ at the initial time. They show the errors in inserting the followers into their desired orbits initially. 

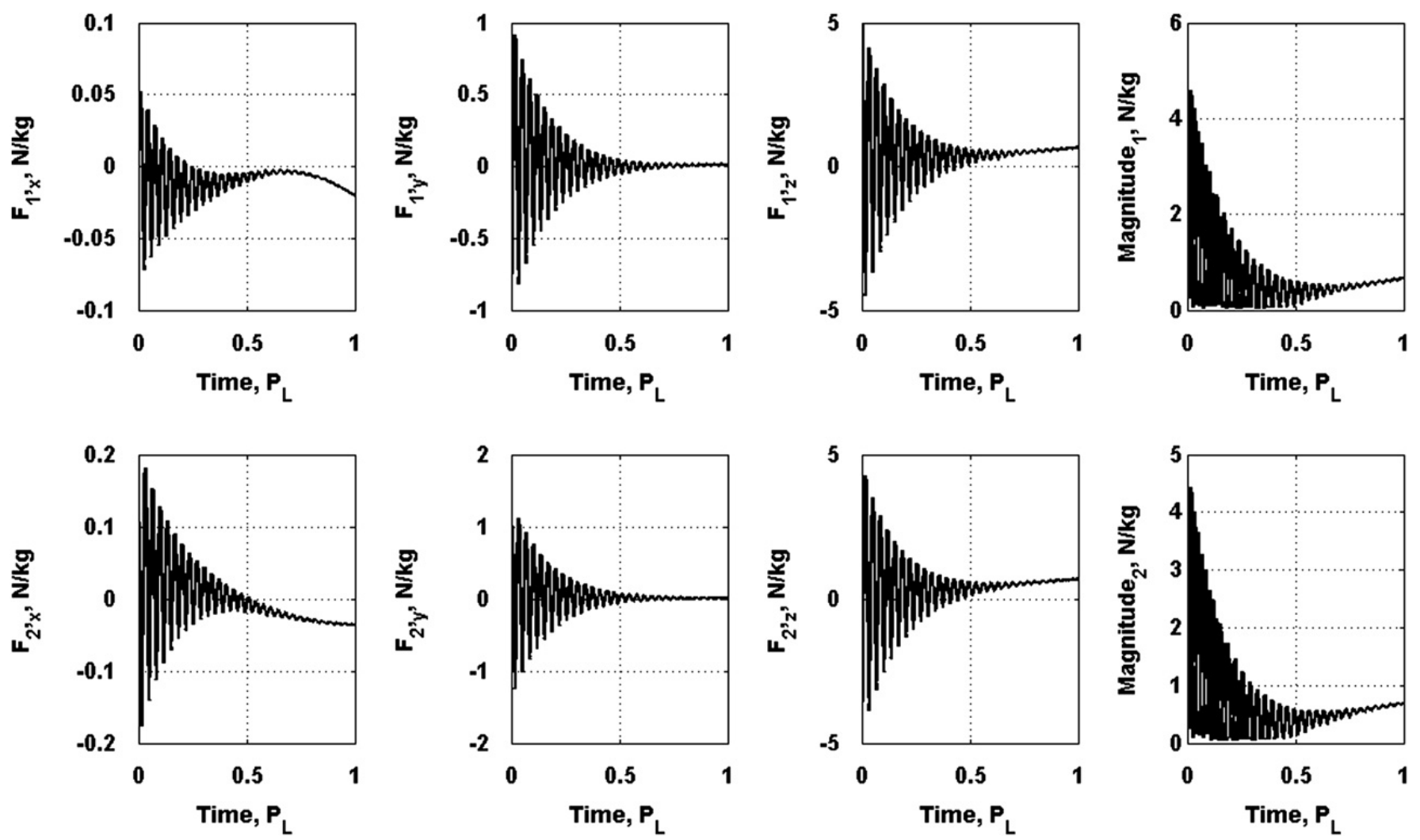

Fig. 19. Required control forces per unit mass in the Hill frame and the total force magnitude over the duration $0-1 P_{L}$ secs. The upper row is for Follower 1 and the lower row is for Follower 2.
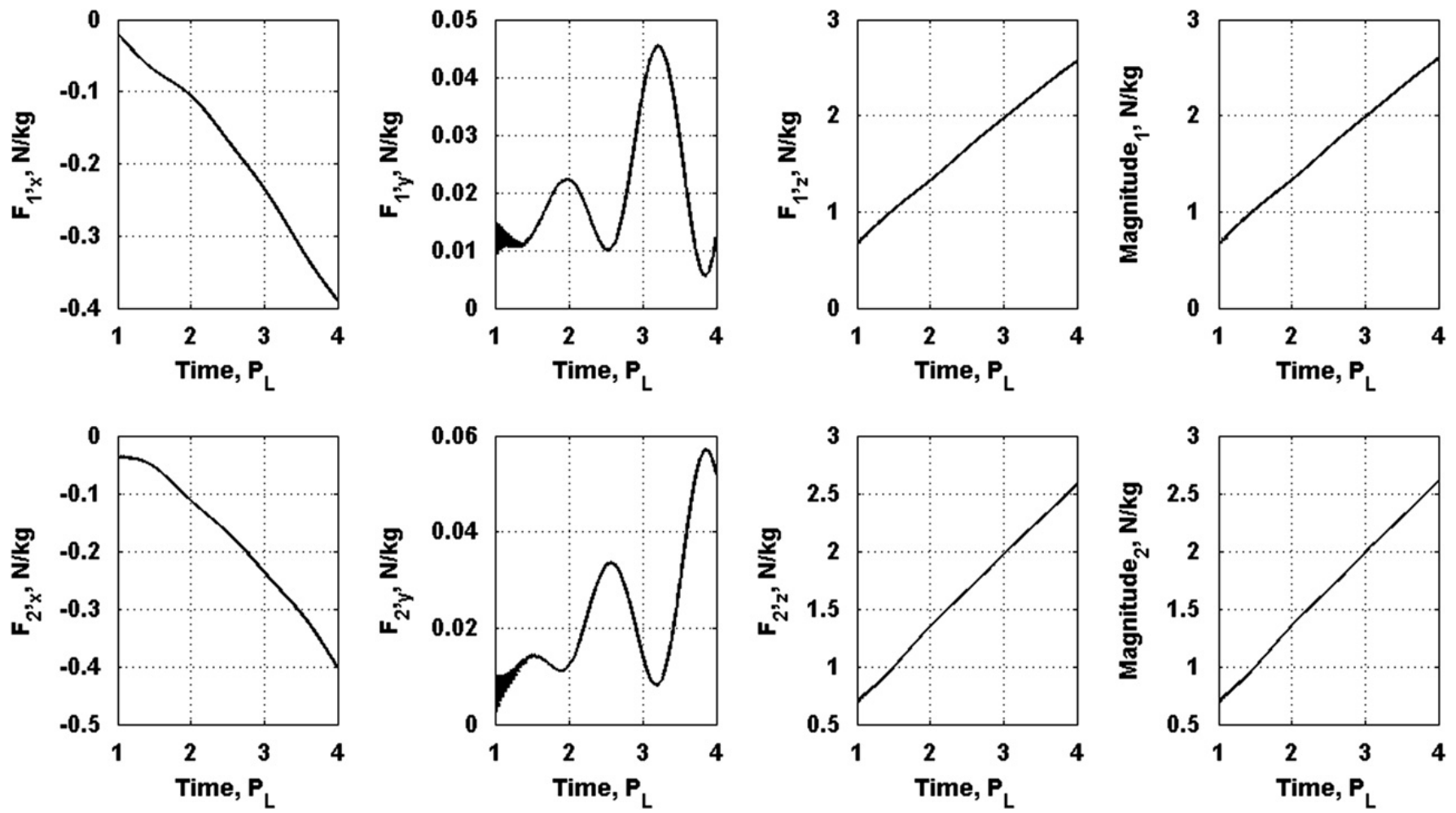

Fig. 20. Required control forces in the Hill frame per unit mass and total force magnitude over the duration $1 P_{L}-4 P_{L}$ secs. The upper row is for Follower 1 and the lower row is for Follower 2.

$\mathbf{M}$ and $\mathbf{A}^{-1}$ are constant and each element of the vector b approaches zero or a sinusoidal, so the effect of $\mathbf{M A}^{-1} \mathbf{b}$ would be less significant than that of Ma, which indicates that the magnitude of the $z$ components of Ma for both followers is relatively large and increasing. 


\section{Conclusion}

In this paper, a new analytic method for the formationkeeping problem is presented. The problem is solved in the Hill frame, which helps us understand the motion more intuitively. The method presented is simple, and provides explicit, closed-form analytical expressions for the control forces needed so as to exactly satisfy the trajectory requirements of the follower(s). Such closedform results for general reference orbits that enable exact control have hereto been unavailable to date. Furthermore, the method is easy to computationally implement, and yields results with high numerical accuracy. The available approaches for formation-keeping to date use linearizations and/or approximations and almost always consider Keplerian reference orbits. Researchers who have considered the entire nonlinear problem, have to date provided only numerical procedures.

The main contributions of the paper are the following.

1. An explicit, closed-form expression for the control force required to exactly satisfy formation-keeping requirements is obtained. Assuming the reference orbit can be represented by an arbitrary (sufficiently smooth) function of time, we get explicitly the closed-form expressions for the control forces required to maintain the given formation configuration precisely. By taking an example of a spiral reference orbit, the ease of implementation and the numerical accuracy of this general methodology are demonstrated.

2. Since the control force to be applied to the follower(s) is explicitly obtained in a closed form and the method is not computationally intensive, it can be easily used for on-orbit, real-time control.

3. The full nonlinear control problem is addressed and no linearizations and/or approximations are made in arriving at the explicit closed form expressions for the control forces.

4. Among all the control forces that cause the system to satisfy the given constraints, the explicit control force obtained herein is optimal in the sense that it minimizes $J(t)=\left(\mathbf{F}^{\mathbf{C}}\right)^{\mathbf{T}} \mathbf{M}^{-1} \mathbf{F}^{\mathbf{C}}$ at each instant of time [21]. Unlike other controllers that use an integral of a weighted norm of the control force over a suitable time duration, the control provided here minimizes $J(t)$ at every instant time.

5. If the reference orbit is a Keplerian orbit, the explicit control to be applied to the follower satellite(s) can be easily and more simply obtained, again yielding a closed-form expression for the control force that ensures exact satisfaction of the constraints imposed on the follower's orbit. Even for this simpler case, the results herein are new since a closed-form expression for the necessary optimal (see item 4 above) control force that is valid for the complete nonlinear problem is found.

6. In practice, it is difficult to initially insert the follower satellite(s) exactly into the desired orbit(s). The problem of finding the necessary control forces when the follower(s) are inserted into orbit(s) with incorrect initial conditions has also been addressed in this paper, and again the explicit closed-form control forces are obtained. We use a stabilization technique to make the errors in the satisfaction of the constraints asymptotically zero and thereby track the desired constraints.

7. Finally, we note that perturbations caused by factors such as the presence of the $J_{2}$ gravitational harmonic, solar pressure, aerodynamic drag, etc., have not been included in this study. However, the methodology developed herein is general, and with a more accurate model that includes these effects the exact control forces needed to be applied to the follower(s) can still be easily obtained in closed form and in a straightforward manner.

8. It is known that some regions of phase space offer better initial conditions than others for the formation-keeping problem [32], in the sense that when starting with initial conditions in these regions the follower(s) maintain their configurations for a longer time with relatively low control energy expenditures. Finding these regions would be an interesting issue for future work.

\section{Appendix}

In this appendix we give explicit expressions for the elements of the matrices $\mathbf{R}, \dot{\mathbf{R}}$, and $\ddot{\mathbf{R}}$. Let us first define the following expressions that will be used frequently:

$$
\begin{aligned}
& r_{L}=\left(X_{L}^{2}+Y_{L}^{2}+Z_{L}^{2}\right)^{1 / 2}, \dot{r}_{L}=\frac{X_{L} \dot{X}_{L}+Y_{L} \dot{Y}_{L}+Z_{L} \dot{Z}_{L}}{r_{L}}, \ddot{r}_{L}=\frac{1}{r_{L}^{2}}\left[\begin{array}{l}
r_{L}\left(\dot{X}_{L}^{2}+\dot{Y}_{L}^{2}+\dot{Z}_{L}^{2}+X_{L} \ddot{X}_{L}+Y_{L} \ddot{Y}_{L}+Z_{L} \ddot{Z}_{L}\right) \\
-\dot{r}_{L}\left(X_{L} \dot{X}_{L}+Y_{L} \dot{Y}_{L}+Z_{L} \dot{Z}_{L}\right)
\end{array}\right], \\
& h_{L}=\left[\left(Y_{L} \dot{Z}_{L}-Z_{L} \dot{Y}_{L}\right)^{2}+\left(Z_{L} \dot{X}_{L}-X_{L} \dot{Z}_{L}\right)^{2}+\left(X_{L} \dot{Y}_{L}-Y_{L} \dot{X}_{L}\right)^{2}\right]^{1 / 2}, \\
& \dot{h}_{L}=\frac{1}{h_{L}}\left[\left(Y_{L} \dot{Z}_{L}-Z_{L} \dot{Y}_{L}\right)\left(Y_{L} \ddot{Z}_{L}-Z_{L} \ddot{Y}_{L}\right)+\left(Z_{L} \dot{X}_{L}-X_{L} \dot{Z}_{L}\right)\left(Z_{L} \ddot{X}_{L}-X_{L} \ddot{Z}_{L}\right)+\left(X_{L} \dot{Y}_{L}-Y_{L} \dot{X}_{L}\right)\left(X_{L} \ddot{Y}_{L}-Y_{L} \ddot{X}_{L}\right)\right],
\end{aligned}
$$


and,

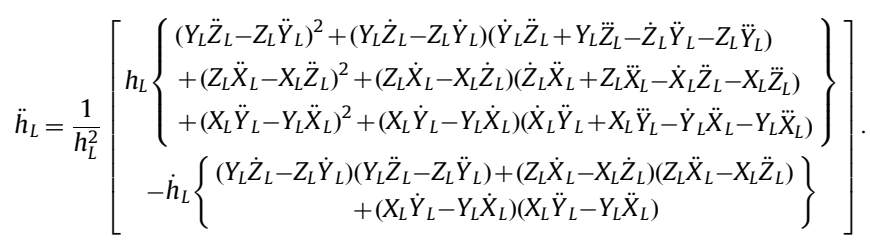

Then, the rotation matrix $\mathbf{R}=\mathbf{S}^{\mathbf{T}}$, and its derivatives $\dot{\mathbf{R}}, \ddot{\mathbf{R}}$ are of the form

$$
\begin{aligned}
\mathbf{R} & =\left[\begin{array}{lll}
R_{11} & R_{12} & R_{13} \\
R_{21} & R_{22} & R_{23} \\
R_{31} & R_{32} & R_{33}
\end{array}\right], \\
\dot{\mathbf{R}} & =\left[\begin{array}{lll}
\dot{R}_{11} & \dot{R}_{12} & \dot{R}_{13} \\
\dot{R}_{21} & \dot{R}_{22} & \dot{R}_{23} \\
\dot{R}_{31} & \dot{R}_{32} & \dot{R}_{33}
\end{array}\right], \\
\ddot{\mathbf{R}} & =\left[\begin{array}{lll}
\ddot{R}_{11} & \ddot{R}_{12} & \ddot{R}_{13} \\
\ddot{R}_{21} & \ddot{R}_{22} & \ddot{R}_{23} \\
\ddot{R}_{31} & \ddot{R}_{32} & \ddot{R}_{33}
\end{array}\right],
\end{aligned}
$$

where each element of these matrices is given as follows:

$$
\begin{aligned}
& R_{11}=\frac{X_{L}}{r_{L}}, \dot{R}_{11}=\frac{r_{L} \dot{X}_{L}-X_{L} \dot{r}_{L}}{r_{L}^{2}}, \\
& \ddot{R}_{11}=\frac{1}{r_{L}^{3}}\left[r_{L}\left(r_{L} \ddot{X}_{L}-X_{L} \ddot{r}_{L}\right)-2 \dot{r}_{L}\left(r_{L} \dot{X}_{L}-X_{L} \dot{r}_{L}\right)\right], \\
& R_{12}=\frac{Y_{L}}{r_{L}} \\
& \dot{R}_{12}=\frac{r_{L} \dot{Y}_{L}-Y_{L} \dot{r}_{L}}{r_{L}^{2}}, \\
& \ddot{R}_{12}=\frac{1}{r_{L}^{3}}\left[r_{L}\left(r_{L} \ddot{Y}_{L}-Y_{L} \ddot{r}_{L}\right)-2 \dot{r}_{L}\left(r_{L} \dot{Y}_{L}-Y_{L} \dot{r}_{L}\right)\right], \\
& R_{13}=\frac{Z_{L}}{r_{L}}, \dot{R}_{13}=\frac{r_{L} \dot{Z}_{L}-Z_{L} \dot{r}_{L}}{r_{L}^{2}}, \\
& \ddot{R}_{13}=\frac{1}{r_{L}^{3}}\left[r_{L}\left(r_{L} \ddot{Z}_{L}-Z_{L} \ddot{r}_{L}\right)-2 \dot{r}_{L}\left(r_{L} \dot{Z}_{L}-Z_{L} \dot{r}_{L}\right)\right], \\
& R_{21}=\frac{1}{h_{L} r_{L}}\left[Z_{L}\left(Z_{L} \dot{X}_{L}-X_{L} \dot{Z}_{L}\right)-Y_{L}\left(X_{L} \dot{Y}_{L}-Y_{L} \dot{X}_{L}\right)\right], \\
& \dot{R}_{21}=\frac{1}{h_{L}^{2} r_{L}^{2}}\left[\begin{array}{c}
h_{L} r_{L}\left\{\dot{Z}_{L}\left(Z_{L} \dot{X}_{L}-X_{L} \dot{Z}_{L}\right)+Z_{L}\left(Z_{L} \ddot{X}_{L}-X_{L} \ddot{Z}_{L}\right)-\dot{Y}_{L}\left(X_{L} \dot{Y}_{L}-Y_{L} \dot{X}_{L}\right)-Y_{L}\left(X_{L} \ddot{Y}_{L}-Y_{L} \ddot{X}_{L}\right)\right\} \\
-\left(\dot{h}_{L} r_{L}+h_{L} \dot{r}_{L}\right)\left\{Z_{L}\left(Z_{L} \dot{X}_{L}-X_{L} \dot{Z}_{L}\right)-Y_{L}\left(X_{L} \dot{Y}_{L}-Y_{L} \dot{X}_{L}\right)\right\}
\end{array}\right],
\end{aligned}
$$

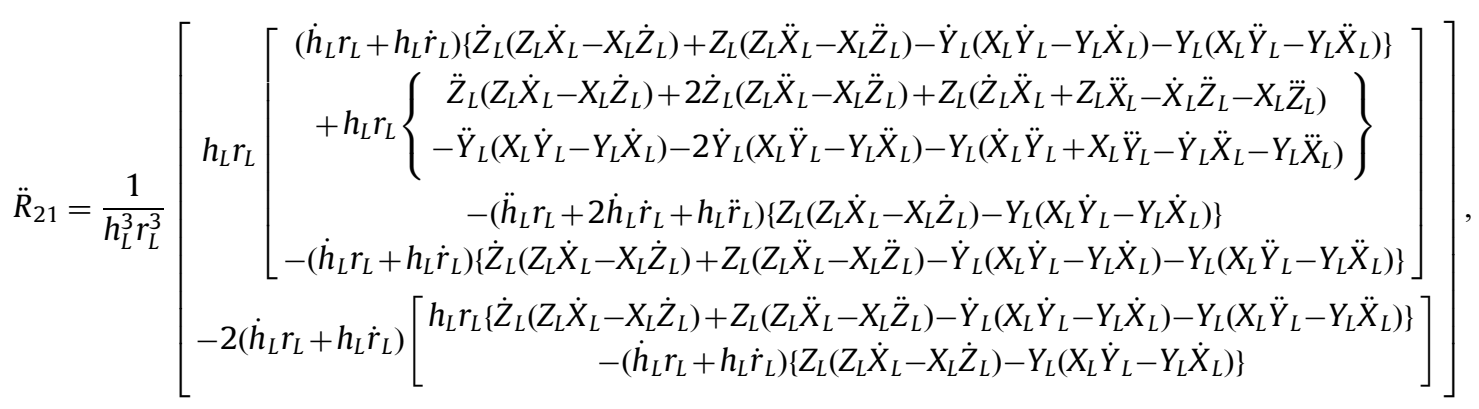

$$
\begin{aligned}
& R_{22}=\frac{1}{h_{L} r_{L}}\left[X_{L}\left(X_{L} \dot{Y}_{L}-Y_{L} \dot{X}_{L}\right)-Z_{L}\left(Y_{L} \dot{Z}_{L}-Z_{L} \dot{Y}_{L}\right)\right], \\
& \dot{R}_{22}=\frac{1}{h_{L}^{2} r_{L}^{2}}\left[\begin{array}{c}
h_{L} r_{L}\left\{\dot{X}_{L}\left(X_{L} \dot{Y}_{L}-Y_{L} \dot{X}_{L}\right)+X_{L}\left(X_{L} \ddot{Y}_{L}-Y_{L} \ddot{X}_{L}\right)-\dot{Z}_{L}\left(Y_{L} \dot{Z}_{L}-Z_{L} \dot{Y}_{L}\right)-Z_{L}\left(Y_{L} \ddot{Z}_{L}-Z_{L} \ddot{Y}_{L}\right)\right\} \\
-\left(\dot{h}_{L} r_{L}+h_{L} \dot{r}_{L}\right)\left\{X_{L}\left(X_{L} \dot{Y}_{L}-Y_{L} \dot{X}_{L}\right)-Z_{L}\left(Y_{L} \dot{Z}_{L}-Z_{L} \dot{Y}_{L}\right)\right\}
\end{array}\right],
\end{aligned}
$$




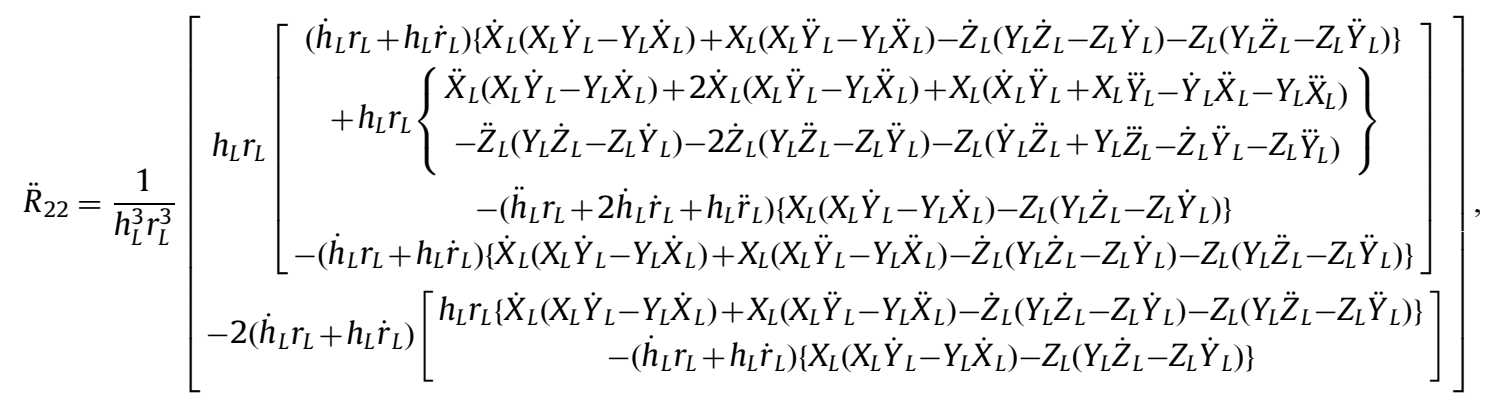$$
R_{23}=\frac{1}{h_{L} r_{L}}\left[Y_{L}\left(Y_{L} \dot{Z}_{L}-Z_{L} \dot{Y}_{L}\right)-X_{L}\left(Z_{L} \dot{X}_{L}-X_{L} \dot{Z}_{L}\right)\right]
$$$$
\dot{R}_{23}=\frac{1}{h_{L}^{2} r_{L}^{2}}\left[\begin{array}{c}
h_{L} r_{L}\left\{\dot{Y}_{L}\left(Y_{L} \dot{Z}_{L}-Z_{L} \dot{Y}_{L}\right)+Y_{L}\left(Y_{L} \ddot{Z}_{L}-Z_{L} \ddot{Y}_{L}\right)-\dot{X}_{L}\left(Z_{L} \dot{X}_{L}-X_{L} \dot{Z}_{L}\right)-X_{L}\left(Z_{L} \ddot{X}_{L}-X_{L} \ddot{Z}_{L}\right)\right\} \\
-\left(\dot{h}_{L} r_{L}+h_{L} \dot{r}_{L}\right)\left\{Y_{L}\left(Y_{L} \dot{Z}_{L}-Z_{L} \dot{Y}_{L}\right)-X_{L}\left(Z_{L} \dot{X}_{L}-X_{L} \dot{Z}_{L}\right)\right\}
\end{array}\right],
$$$$
\ddot{R}_{23}=\frac{1}{h_{L}^{3} r_{L}^{3}}\left[\begin{array}{c}
h_{L} r_{L}\left[\begin{array}{c}
\left(\dot{h}_{L} r_{L}+h_{L} \dot{r}_{L}\right)\left\{\dot{Y}_{L}\left(Y_{L} \dot{Z}_{L}-Z_{L} \dot{Y}_{L}\right)+Y_{L}\left(Y_{L} \ddot{Z}_{L}-Z_{L} \ddot{Y}_{L}\right)-\dot{X}_{L}\left(Z_{L} \dot{X}_{L}-X_{L} \dot{Z}_{L}\right)-X_{L}\left(Z_{L} \ddot{X}_{L}-X_{L} \ddot{Z}_{L}\right)\right\} \\
+h_{L} r_{L}\left\{\begin{array}{c}
\ddot{Y}_{L}\left(Y_{L} \dot{Z}_{L}-Z_{L} \dot{Y}_{L}\right)+2 \dot{Y}_{L}\left(Y_{L} \ddot{Z}_{L}-Z_{L} \ddot{Y}_{L}\right)+Y_{L}\left(\dot{Y}_{L} \ddot{Z}_{L}+Y_{L} \ddot{Z}_{L}-\dot{Z}_{L} \ddot{Y}_{L}-Z_{L} \ddot{Y}_{L}\right) \\
-\ddot{X}_{L}\left(Z_{L} \dot{X}_{L}-X_{L} \dot{Z}_{L}\right)-2 \dot{X}_{L}\left(Z_{L} \ddot{X}_{L}-X_{L} \ddot{Z}_{L}\right)-X_{L}\left(\dot{Z}_{L} \ddot{X}_{L}+Z_{L} \ddot{X}_{L}-\dot{X}_{L} \ddot{Z}_{L}-X_{L} \ddot{Z}_{L}\right)
\end{array}\right\} \\
-\left(\ddot{h}_{L} r_{L}+2 \dot{h}_{L} \dot{r}_{L}+h_{L} \ddot{r}_{L}\right)\left\{Y_{L}\left(Y_{L} \dot{Z}_{L}-Z_{L} \dot{Y}_{L}\right)-X_{L}\left(Z_{L} \dot{X}_{L}-X_{L} \dot{Z}_{L}\right)\right\} \\
-\left(\dot{h}_{L} r_{L}+h_{L} \dot{r}_{L}\right)\left\{\dot{Y}_{L}\left(Y_{L} \dot{Z}_{L}-Z_{L} \dot{Y}_{L}\right)+Y_{L}\left(Y_{L} \ddot{Z}_{L}-Z_{L} \ddot{Y}_{L}\right)-\dot{X}_{L}\left(Z_{L} \dot{X}_{L}-X_{L} \dot{Z}_{L}\right)-X_{L}\left(Z_{L} \ddot{X}_{L}-X_{L} \ddot{Z}_{L}\right)\right\}
\end{array}\right] \\
-2\left(\dot{h}_{L} r_{L}+h_{L} \dot{r}_{L}\right)\left[\begin{array}{c}
h_{L} r_{L}\left(\dot{Y}_{L}\left(Y_{L} \dot{Z}_{L}-Z_{L} \dot{Y}_{L}\right)+Y_{L}\left(Y_{L} \ddot{Z}_{L}-Z_{L} \ddot{Y}_{L}\right)-\dot{X}_{L}\left(Z_{L} \dot{X}_{L}-X_{L} \dot{Z}_{L}\right)-X_{L}\left(Z_{L} \ddot{X}_{L}-X_{L} \ddot{Z}_{L}\right)\right\} \\
-\left(\dot{h}_{L} r_{L}+h_{L} \dot{r}_{L}\right)\left\{Y_{L}\left(Y_{L} \dot{Z}_{L}-Z_{L} \dot{Y}_{L}\right)-X_{L}\left(Z_{L} \dot{X}_{L}-X_{L} \dot{Z}_{L}\right)\right\}
\end{array}\right]
\end{array}\right],
$$

$$
R_{31}=\frac{Y_{L} \dot{Z}_{L}-Z_{L} \dot{Y}_{L}}{h_{L}}, \dot{R}_{31}=\frac{1}{h_{L}^{2}}\left[h_{L}\left(Y_{L} \ddot{Z}_{L}-Z_{L} \ddot{Y}_{L}\right)-\dot{h}_{L}\left(Y_{L} \dot{Z}_{L}-Z_{L} \dot{Y}_{L}\right)\right]
$$$$
\ddot{R}_{31}=\frac{1}{h_{L}^{3}}\left[\begin{array}{c}
h_{L}\left\{h_{L}\left(\dot{Y}_{L} \ddot{Z}_{L}+Y_{L} \ddot{Z}_{L}-\dot{Z}_{L} \ddot{Y}_{L}-Z_{L} \ddot{Y}_{L}\right)-\ddot{h}_{L}\left(Y_{L} \dot{Z}_{L}-Z_{L} \dot{Y}_{L}\right)\right\} \\
-2 \dot{h}_{L}\left\{h_{L}\left(Y_{L} \ddot{Z}_{L}-Z_{L} \ddot{Y}_{L}\right)-\dot{h}_{L}\left(Y_{L} \dot{Z}_{L}-Z_{L} \dot{Y}_{L}\right)\right\}
\end{array}\right],
$$$$
R_{32}=\frac{Z_{L} \dot{X}_{L}-X_{L} \dot{Z}_{L}}{h_{L}}, \dot{R}_{32}=\frac{1}{h_{L}^{2}}\left[h_{L}\left(Z_{L} \ddot{X}_{L}-X_{L} \ddot{Z}_{L}\right)-\dot{h}_{L}\left(Z_{L} \dot{X}_{L}-X_{L} \dot{Z}_{L}\right)\right],
$$$$
\ddot{R}_{32}=\frac{1}{h_{L}^{3}}\left[\begin{array}{c}
h_{L}\left\{h_{L}\left(\dot{Z}_{L} \ddot{X}_{L}+Z_{L} \ddot{X}_{L}-\dot{X}_{L} \ddot{Z}_{L}-X_{L} \ddot{Z}_{L}\right)-\ddot{h}_{L}\left(Z_{L} \dot{X}_{L}-X_{L} \dot{Z}_{L}\right)\right\} \\
-2 \dot{h}_{L}\left\{h_{L}\left(Z_{L} \ddot{X}_{L}-X_{L} \ddot{Z}_{L}\right)-\dot{h}_{L}\left(Z_{L} \dot{X}_{L}-X_{L} \dot{Z}_{L}\right)\right\}
\end{array}\right],
$$$$
R_{33}=\frac{X_{L} \dot{Y}_{L}-Y_{L} \dot{X}_{L}}{h_{L}}, \dot{R}_{33}=\frac{1}{h_{L}^{2}}\left[h_{L}\left(X_{L} \ddot{Y}_{L}-Y_{L} \ddot{X}_{L}\right)-\dot{h}_{L}\left(X_{L} \dot{Y}_{L}-Y_{L} \dot{X}_{L}\right)\right]
$$

and

$$
\ddot{R}_{33}=\frac{1}{h_{L}^{3}}\left[\begin{array}{c}
h_{L}\left\{h_{L}\left(\dot{X}_{L} \ddot{Y}_{L}+X_{L} \ddot{Y}_{L}-\dot{Y}_{L} \ddot{X}_{L}-Y_{L} \ddot{X}_{L}\right)-\ddot{h}_{L}\left(X_{L} \dot{Y}_{L}-Y_{L} \dot{X}_{L}\right)\right\} \\
-2 \dot{h}_{L}\left\{h_{L}\left(X_{L} \ddot{Y}_{L}-Y_{L} \ddot{X}_{L}\right)-\dot{h}_{L}\left(X_{L} \dot{Y}_{L}-Y_{L} \dot{X}_{L}\right)\right\}
\end{array}\right] .
$$

\section{References}

[1] D.P. Scharf, F.Y. Hadaegh, S.R. Ploen, A survey of spacecraft formation flying guidance and control (part I): guidance, in: Proceedings of American Control Conference, Denver, CO, June 2003, pp. 1733-1739.

[2] D.P. Scharf, F.Y. Hadaegh, S.R. Ploen, A survey of spacecraft formation flying guidance and control (part II): control, in: Proceedings of American Control Conference, Boston, MA, June 2004, pp. 2976-2985.

[3] M. Tillerson, G. Inalhan, J.P. How, Co-ordination and control of distributed spacecraft systems using convex optimization techniques, Int. J. Robust Nonlin. 12 (2002) 207-242.

[4] G.W. Hill, Researches in the lunar theory, Am. J. Math. 1 (1) (1878) 5-26.
[5] W.H. Clohessy, R.S. Wiltshire, Terminal guidance system for satellite rendezvous, J. Aerosp. Sci. 27 (8) (1960) 653-658 674.

[6] J. Tschauner, P. Hempel, Rendezvous zu Einemin Elliptischer Bahn Umlaufenden Ziel, Astronaut. Acta 11 (2) (1965) 104-109.

[7] Q. Yan, V. Kapila, A.G. Sparks, Pulse-based periodic control for spacecraft formation flying, in: Proceedings of American Control Conference, Chicago, IL, June 2000, pp. 374-378.

[8] A. Sparks, Satellite formationkeeping control in the presence of gravity perturbations, in: Proceedings of American Control Conference, Chicago, IL, June 2000, pp. 844-848.

[9] G. Inalhan, M. Tillerson, J.P. How, Relative dynamics and control of spacecraft formations in eccentric orbits, J. Guidance Control Dyn. 25 (1) (2002) 48-59. 
[10] M. Tillerson, J.P. How, Advanced guidance algorithms for spacecraft formation-keeping, in: Proceedings of American Control Conference, Anchorage, AK, May 2002, pp. 2830-2835.

[11] M. Qingsong, W. Pengji, Y. Di, Low-thrust fuzzy formation keeping for multiple spacecraft flying, Acta Astronaut. 55 (11) (2004) 895-901.

[12] F.Y. Hadaegh, W.-M. Lu, P.K.C. Wang, Adaptive control of formation flying spacecraft for interferometry, in: Proceedings of International Federation of Automatic Control, 1998, pp. 195-200.

[13] M.B. Milam, N. Petit, R.M. Murray, Constrained trajectory generation for micro-satellite formation flying, in: AIAA Guidance, Navigation, and Control Conference and Exhibit, Paper AIAA 2001-4030, Montreal, Canada, August 2001.

[14] T.S. No, J.G. Lee, J.E. Cochran Jr., Spacecraft formation-keeping using a closed-form orbit propagator and optimization technique, Acta Astronaut. 65 (4) (2009) 537-548.

[15] F.E. Udwadia, R.E. Kalaba, What is the general form of the explicit equations of motion for constrained mechanical systems, J. Appl. Mech. 69 (3) (2002) 335-339.

[16] F.E. Udwadia, Equations of motion for mechanical systems: a unified approach, Int. J. Nonlin. Mech. 31 (6) (1996) 951-958.

[17] F.E. Udwadia, Nonideal constraints and Lagrangian dynamics, J. Aerosp. Eng. 13 (1) (2000) 17-22.

[18] R.E. Kalaba, F.E. Udwadia, Equations of motion for nonholonomic, constrained dynamical systems via Gauss's principle, J. Appl. Mech. 60 (3) (1993) 662-668.

[19] F.E. Udwadia, A new perspective on the tracking control of nonlinear structural and mechanical systems, Proc. R. Soc. London A 459 (2003) 1783-1800.

[20] F.E. Udwadia, Equations of motion for constrained multibody systems and their control, J. Optimiz. Theory App. 127 (3) (2005) 627-638.
[21] F.E. Udwadia, Optimal tracking control of nonlinear dynamical systems, Proc. R. Soc. London A 464 (2008) 2341-2363.

[22] C.F. Gauss, Uber ein neues allgemeines Grundgsetz der Mechanik, J. Reine Ang. Math. 4 (1829) 232-235.

[23] A.D. Schutte, B.A. Dooley, Constrained motion of tethered satellites, J. Aerosp. Eng. 18 (4) (2005) 242-250.

[24] T. Lam, New approach to mission design based on the fundamental equations of motion, J. Aerosp. Eng. 19 (2) (2006) 59-67.

[25] T. Lam, A. Schutte, F.E. Udwadia, Constraint based control method for precision formation flight of spacecraft, in: Proceedings of AAS/ AIAA Space Flight Mechanics Meeting, Paper AAS 06-122, Tampa, Florida, January 2006.

[26] H. Cho, A. Yu, New approach to satellite formation-keeping: exact solution to the full nonlinear problem, J. Aerosp. Eng. 22 (4) (2009) $445-455$.

[27] C. Sabol, R. Burns, C.A. McLaughlin, Satellite formation flying design and evolution, J. Spacecr. Rockets 38 (2) (2001) $270-278$

[28] F.E. Udwadia, R.E. Kalaba, Analytical Dynamics: A New Approach, Cambridge University Press, New York, 2008.

[29] J.E. Prussing, B.A. Conway, Orbital Mechanics, Oxford University Press, New York, 1993.

[30] D.A. Vallado, Fundamentals of Astrodynamics and Applications, second ed., The Space Technology Library, El Segundo, 2001.

[31] J. Baumgarte, Stabilization of constraints and integrals of motion in dynamical systems, Comput. Method Appl. M. 1 (1) (1972) $1-16$.

[32] W.S. Koon, J.E. Marsden, J. Masdemont, R.M. Murray, $J_{2}$ dynamics and formation flight, in: Proceedings of AIAA Guidance, Navigation, and Control Conference, Paper AIAA 2001-4090, Montreal, Canada, August 2001. 